\title{
GENERICALLY ALGEBRAIC ALGEBRAS
}

\author{
BY \\ KEVIN MCCRIMMON( ${ }^{(1)}$
}

The notions of generic minimum polynomial and generic norm for finitedimensional strictly power-associative algebras were introduced by Professor $\mathbf{N}$. Jacobson in [2], [3], generalizing the notions of principal polynomial and norm for associative algebras. In this paper we extend these concepts to infinite-dimensional algebras; in the process we give a coordinate-free approach to the generic norm.

In the first section we review the machinery of the differential calculus in infinite-dimensional spaces, including the definition of rational mappings, the Zariski topology, and differential operators. In the second section we establish the result, which is fundamental in the sequel, that every homogeneous polynomial function can be written uniquely as a product of a finite number of irreducible polynomial functions. We then apply this factorization theory to show that under certain general conditions the factors of an automorphic form (relative to a group of linear transformations) are again automorphic forms.

The third and fourth sections are devoted to defining and establishing the basic properties of the generic minimum polynomial and generic norm. A generically algebraic algebra is defined as one which is "uniformly" algebraic in the sense that each element $x$ satisfies a monic polynomial $m_{x}(\lambda)$ which varies "continuously" as a function of $x: m_{x}(x)=0$ for $m_{x}(\lambda)=\sum m_{\imath}(x) \lambda^{\lambda}$ where the coefficients are polynomial functions. The generic minimum polynomial is the polynomial of least degree having these properties, and the generic norm is plus or minus its constant term. Using the factorization theorem the standard properties of the generic norm carry over easily.

The fifth section discusses the discriminant of an algebra. An algebra is called unramified if its discriminant is not identically zero. Intuitively, this means that the algebra modulo or radical is separable. Our definition is motivated by [1, p. 105]; it differs from that given in [2].

Several investigations revealed a close connection between the generic norm and forms admitting composition [4], [5], [6], [7], [8]. A general conjecture of Professor R. D. Schafer was that every norm on a normed algebra was a product of irreducible factors of the generic norm. This was known for finite-dimensional algebras [4] and for infinite-dimensional algebras in certain special cases [5]. In the sixth section of the paper we apply our results to settle the general case.

Received by the editors June 6, 1966.

(1) This research was supported by the Air Force Office of Scientific Research through an NAS-NRC Postdoctoral Research Fellowship. 
The final part of the paper is devoted to briefly indicating how the novel approach of H. Braun and M. Koecher to nonassociative algebras [1] can be carried over to this infinite-dimensional setting. Homogeneous algebras are defined geometrically as those whose structure group acts transitively on the set of nonsingular elements over a suitable extension field. The Equivalence Theorem shows that homogeneity and several other geometric conditions are equivalent to the condition that the symmetrized algebra be Jordan. Several properties of the reduced norm for a homogeneous algebra are then derived.

The structure theory is approached by means of associative bilinear forms. The nondegeneracy conditions for an algebra require that there be enough associative forms to separate points. Use of these forms considerably facilitates the proofs of the standard theorems. The final structure theorem shows that the classes of simple algebras obtained are essentially the normed algebras-the quasiassociative algebras, commutative Jordan algebras, and algebras of degree 2.

1. The differential calculus. Throughout this paper $\mathfrak{X}, \mathfrak{Y}, \mathbf{3}$ will denote vector spaces, not necessarily finite-dimensional, over an infinite field $\Phi$. We review the results of [5, pp. 73-75].

$\left\{\mathfrak{X}_{\alpha}\right\}$ will denote the collection of finite-dimensional subspaces of $\mathfrak{X}$. Each such subspace has a natural topology, the Zariski topology. If $\mathfrak{X}_{\alpha} \subset \mathfrak{X}_{\beta}$ the Zariski topology on $\mathfrak{X}_{\alpha}$ is just the topology induced by $\mathfrak{X}_{\beta}$, so the inclusion map $\iota_{\beta \alpha}$ is continuous. Since the $\left\{\mathfrak{X}_{\alpha}\right\}$ form a directed set, $\mathfrak{X}$ is in a natural way the direct limit of the topological spaces $\mathfrak{X}_{\alpha}$. The induced topology on $\mathfrak{X}$ is again called the Zariski topology. Thus $V$ is open in $\mathfrak{X}$ if and only if $V \cap \mathfrak{X}_{\alpha}$ is open in $\mathfrak{X}_{\alpha}$ for each $\alpha$. As in the finite-dimensional case, nonempty open sets are dense.

If $\mathfrak{Y}$ is another vector space we have for each $\alpha$ spaces $\mathscr{P}\left(\mathfrak{X}_{\alpha}, \mathfrak{Y}\right)$ and $\mathscr{R}\left(\mathfrak{X}_{\alpha}, \mathfrak{Y}\right)$ of polynomial or rational mappings of $\mathfrak{X}_{\alpha}$ or an open subset of $\mathfrak{X}_{\alpha}$ into $\mathfrak{Y}$; these spaces are spanned by the mappings $F_{\alpha}: x_{\alpha} \rightarrow f_{\alpha}\left(x_{\alpha}\right) y$ where $y \in \mathfrak{Y}$ and $f_{\alpha}$ is an ordinary polynomial or rational function on $\mathfrak{X}_{\alpha}$. Note in particular that the range $F_{\alpha}\left(\mathfrak{X}_{\alpha}\right)$ of any $F_{\alpha} \in \mathscr{R}\left(\mathfrak{X}_{\alpha}, \mathfrak{Y}\right)$ is contained in a finite-dimensional subspace $\mathfrak{Y}_{\alpha}$ of $\mathfrak{Y}$. If $\mathfrak{X}_{\alpha} \subset \mathfrak{X}_{\beta}$ we have the natural restriction $\rho_{\alpha \beta}=\iota_{\beta \alpha}^{*}$ of $\mathscr{P}\left(\mathfrak{X}_{\beta}, \mathfrak{Y}\right) \rightarrow \mathscr{P}\left(\mathfrak{X}_{\alpha}, \mathfrak{Y}\right)$ or $\mathscr{R}\left(\mathfrak{X}_{\beta}, \mathfrak{Y}\right) \rightarrow \mathscr{R}\left(\mathfrak{X}_{\alpha}, \mathfrak{Y}\right)$, and we may form the inverse limits $\mathscr{P}(\mathfrak{X}, \mathfrak{Y})$ or $\mathscr{R}(\mathfrak{X}, \mathfrak{Y})$ of polynomial or rational mappings of $\mathfrak{X}$ or an open subset of $\mathfrak{X}$ into $\mathfrak{Y}$. For $\mathfrak{Y}=\Phi$, $\mathscr{P}(\mathfrak{X})=\mathscr{P}(\mathfrak{X}, \Phi)$ and $\mathscr{R}(\mathfrak{X})=\mathscr{R}(\mathfrak{X}, \Phi)$ are the polynomial or rational functions on $\mathfrak{X}$. A (set-theoretic) mapping $F: \mathfrak{X} \rightarrow \mathfrak{Y}$ defined on some open subset of $\mathfrak{X}$ is in $\mathscr{P}(\mathfrak{X}, \mathfrak{Y})$ or $\mathscr{R}(\mathfrak{X}, \mathfrak{Y})$ if and only if each restriction $F_{\alpha}=\left.F\right|_{\mathfrak{x}_{\alpha}}$ is in $\mathscr{P}\left(\mathfrak{X}_{\alpha}, \mathfrak{Y}\right)$ or $\mathscr{R}\left(\mathfrak{X}_{\alpha}, \mathfrak{Y}\right)$. Thus $F$ is pieced together from a consistent family $\left\{F_{\alpha}\right\}$ of local functions. It is useful to observe that $F$ can be formed from a consistent family $\left\{F_{\beta}\right\}$ of functions on any cofinal collection $\left\{\mathfrak{X}_{\beta}\right\}$ of subspaces.

Unfortunately, for infinite-dimensional spaces the Zariski topology is strictly finer than that generated by the open sets $V(F)=\{x \in \mathfrak{X} \mid F(x) \neq 0\}$ for $F \in \mathscr{P}(\mathfrak{X})$, and $\mathscr{R}(\mathfrak{X})$ is no longer the quotient field of $\mathscr{P}(\mathfrak{X})$. For example, if $\left\{x_{1}, x_{2}, \ldots\right\}$ is a basis for $\mathfrak{X}$ set $F_{n}\left(\xi_{1}, \ldots, \xi_{n}\right)=\xi_{1}^{-n}\left(\xi_{1}-\xi_{2}\right) \cdots\left(\xi_{1}-\xi_{n}\right)$ on the subspace $\mathfrak{X}_{n}$ 
$=\left\{x_{1}, \ldots, x_{n}\right\}$; these determine a rational function $F$ which is not the quotient of two polynomials since $\{x \in \mathfrak{X} \mid F(x)$ is zero or is undefined $\}$ is a closed set on which no nonzero polynomial vanishes. Actually, the topology and the exact nature of the mappings plays very little role in our theory.

We regard $\mathscr{R}(\mathfrak{X})$ as a space of (set-theoretic) functions, but at times we will also want to regard it as a field. The situation is analogous to that in the theory of functions of a real variable, where at times one regards the objects under study as functions and at times as equivalence classes of functions which differ only on sets of measure zero. We can regard two rational functions as equivalent if they are defined and equal on some dense open set. Each equivalence class has a unique representative with maximal domain of definition, and considering only such "maximal" functions the usual relations such as $F \cdot F^{-1}=1$ are valid.

All the machinery of the differential calculus can be carried over to this setting. For $x \in \mathfrak{X}, F \in \mathscr{R}(\mathfrak{X}, \mathfrak{Y})$ the differential of $F$ at $x$ is a linear map $\left.\partial F\right|_{x}: \mathfrak{X} \rightarrow \mathfrak{Y}$ whose value at a vector $u \in \mathfrak{X}$ is $\left.\partial_{u} F\right|_{x}=\left.\partial F\right|_{x}(u) . x \rightarrow \partial_{u} F(x)$ is a rational map $\partial_{u} F: \mathfrak{X} \rightarrow \mathfrak{Y}$ and $F \rightarrow \partial_{u} F$ is just partial derivation $\partial_{u}$ in the direction $u . \partial F:\left.x \rightarrow \partial F\right|_{x}$ is a settheoretic mapping from $\mathfrak{X}$ to $\operatorname{Hom}(\mathfrak{X}, \mathfrak{Y})$, but it need not be rational.

If $F \in \mathscr{R}(\mathfrak{Y}, \mathbb{3}), G \in \mathscr{R}(\mathfrak{X}, \mathfrak{Y})$ then $F \circ G \in \mathscr{R}(\mathfrak{X}, \mathbb{Z})$ and the chain rule implies

$$
\begin{aligned}
\left.\partial\{F \circ G\}\right|_{x} & =\left.\left.\partial F\right|_{G(x)} \circ \partial G\right|_{x}, \\
\left.\partial_{u}\{F \circ G\}\right|_{x} & =\left.\partial_{v} F\right|_{G(x)}, v=\left.\partial_{u} G\right|_{x} .
\end{aligned}
$$

For rational functions we can define the logarithmic derivative

$$
\partial_{u} \log F=F^{-1} \partial_{u} F
$$

with the usual rules

$$
\begin{gathered}
\partial \log F \cdot G=\partial \log F+\partial \log G, \\
\partial_{u} \partial_{v} \log F=\partial_{v} \partial_{u} \log F=F^{-2}\left\{F \cdot \partial_{u} \partial_{v} F-\partial_{u} F \cdot \partial_{v} F\right\} .
\end{gathered}
$$

$F$ is inseparable if $\partial F=0$; this means that on any $\mathfrak{X}_{\alpha}=\left\{x_{1}, \ldots, x_{n}\right\}$ we have $F_{\alpha}\left(\xi_{1}, \ldots, \xi_{n}\right)=G_{\alpha}\left(\xi_{1}^{p}, \ldots, \xi_{n}^{p}\right)$ where $p$ denotes the characteristic of $\Phi$. If $\Phi$ is perfect this is equivalent to $F=G^{p}$ for some rational function $G$. $F$ is homogeneous of degree $n$ if $F(\lambda x)=\lambda^{n} F(x)$ for all $\lambda \in \Phi$; a homogeneous polynomial function is called a form. Finally, note that any $F \in \mathscr{R}(\mathfrak{X}, \mathfrak{Y})$ has a unique extension $F_{\Omega}$ $\in \mathscr{R}\left(\mathfrak{X}_{\Omega}, \mathfrak{Y}_{\Omega}\right)$ for any extension $\Omega$ of the base field $\Phi$.

2. Factorization of forms. A polynomial function $P$ divides or is a factor of $Q$ if there is a polynomial function $R$ with $Q=P R$; equivalently, $Q / P$ is a polynomial function (on each finite-dimensional subspace). $P$ is irreducible if it has no proper factors, and prime if it divides either $R$ or $S$ whenever it divides their product $R S$. Throughout the rest of this paper $P, Q, R, S$ will denote forms of positive degree $p, q, r, s>0$. Note that all factors of a form are again forms, since this is true locally. 
Suppose $\mathfrak{X}_{\alpha} \subset \mathfrak{X}_{\beta}$ are two finite-dimensional subspaces. Observe that if $\boldsymbol{P}_{\beta}$ is a form on $\mathfrak{X}_{\beta}$ then its restriction $P_{\alpha}$ to $\mathfrak{X}_{\alpha}$ is again a form of the same degree and hence either nonconstant or identically zero. If $P_{\beta}$ is prime on $\mathfrak{X}_{\beta}$ then $P_{\alpha}$ may not be prime on $\mathfrak{X}_{\alpha}$, in which case we say $P_{\beta}$ splits upon restriction to $\mathfrak{X}_{\alpha}$; if $P_{\beta}, R_{\beta}$ are distinct primes on $\mathfrak{X}_{\beta}$ they may still be prime on $\mathfrak{X}_{\alpha}$ but not distinct, $P_{\alpha}=R_{\alpha}$, in which case we say they coalesce on restriction to $\mathfrak{X}_{\alpha}$. If $x_{0}$ is a point in $\mathfrak{X}_{\alpha}$ where $Q\left(x_{0}\right) \neq 0, Q_{\alpha}=Q\left(x_{0}\right) \prod Q_{i \alpha}^{q_{i \alpha}}$ and $Q_{\beta}=Q\left(x_{0}\right) \prod Q_{i \beta}^{q_{i \beta}}$ the (unique) factorizations of $Q$ on $\mathfrak{X}_{\alpha}$ and $\mathfrak{X}_{\beta}$ into distinct prime factors $Q_{i \alpha}$ and $Q_{i \beta}$ with $Q_{i \alpha}\left(x_{0}\right)=Q_{i \beta}\left(x_{0}\right)=1$, then $\mathfrak{X}_{\beta}$ is a stable extension of $\mathfrak{X}_{\alpha}$ for $Q$ if there is no splitting or coalescing among the $Q_{i \beta}$ upon restriction. $\mathfrak{X}_{\alpha}$ is a stability space for $Q$ if all extensions $\mathfrak{X}_{\beta}$ of $\mathfrak{X}_{\alpha}$ are stable. Note that any extension of a stability space is again a stability space.

LEMMA 1. Every form $Q$ has a stability space.

Proof. Fix a point $x_{0}$ where $Q\left(x_{0}\right) \neq 0$, and consider only spaces $\mathfrak{X}_{\alpha}$ containing $x_{0}$. If

$$
Q_{\alpha}=Q\left(x_{0}\right) \prod_{i=1}^{n_{\alpha}} Q_{i \alpha}^{q_{i \alpha}}
$$

is the unique factorization of $Q_{\alpha}$ with $Q_{i \alpha}\left(x_{0}\right)=1, q_{i \alpha}>0$, let

$$
m_{\alpha}=\sum_{i=1}^{n_{\alpha}} q_{i \alpha}
$$

be the total number of prime factors. We claim that if $\mathfrak{X}_{\alpha} \subset \mathfrak{X}_{\beta}$ then

Indeed, if

$$
m_{\alpha} \geqq m_{\beta} .
$$

$$
Q_{\beta}=Q\left(x_{0}\right) \prod_{i=1}^{n_{\beta}} Q_{i \beta}^{q_{i \beta}}
$$

is the corresponding factorization on $\mathfrak{X}_{\beta}$ then the restriction of $Q_{i \beta}$ to $\mathfrak{X}_{\alpha}$ is nonconstant, hence is prime or splits into several factors. Thus the total number of factors of the restriction of $Q_{\beta}$ to $\mathfrak{X}_{\alpha}$ is at least $m_{\beta}$; this restriction is $Q_{\alpha}$ and this total number is $m_{\alpha}$ by the uniqueness of the decomposition on $\mathfrak{X}_{\alpha}$, so the above inequality holds. We have equality only if no splitting occurs among the $Q_{i \beta}$ upon restriction.

Choose $\mathfrak{X}_{\alpha}$ with minimal $m_{\alpha}$. By the above, for any extension $\mathfrak{X}_{\beta}$ we have $m_{\alpha}=m_{\beta}$ and no splitting occurs upon restriction of the primes $Q_{i \beta}$. In this case we claim

$$
n_{\alpha} \leqq n_{\beta} \text {. }
$$

Indeed, the restrictions of $Q_{i \beta}$ to $\mathfrak{X}_{\alpha}$ remain prime but need not be distinct, so the number of distinct primes appearing is at most $n_{\beta}$; the number of distinct factors of $Q_{\alpha}$ is $n_{\alpha}$, so the above inequality holds. We have equality only if none of the $Q_{i \beta}$ coalesce upon restriction to $\mathfrak{X}_{\alpha}$. 
Finally, among the spaces $\mathfrak{X}_{\alpha}$ with minimal $m_{\alpha}$ choose one with maximal $n_{\alpha}$. Then for $\mathfrak{X}_{\alpha} \subset \mathfrak{X}_{\beta}$ we have $m_{\alpha}=m_{\beta}, n_{\alpha}=n_{\beta}$ and hence no splitting or coalescing appears upon restriction of the $Q_{i \beta}$ to $\mathfrak{X}_{\alpha}$, all $\mathfrak{X}_{\beta}$ are stable extensions of $\mathfrak{X}_{\alpha}$, and $\mathfrak{X}_{\alpha}$ is a stability space for $Q$.

THEOREM 1. If $Q$ is a form, $x_{0}$ a point with $Q\left(x_{0}\right) \neq 0$, then $Q$ has a unique factorization $Q=Q\left(x_{0}\right) \prod Q_{i}^{q_{i}}$ into irreducible factors $Q_{i}$ with $Q_{i}\left(x_{0}\right)=1$.

Proof. Let $\mathfrak{X}_{\alpha}$ be a stability space for $Q$ containing $x_{0}, Q_{\alpha}=Q\left(x_{0}\right) \prod Q_{i \alpha}^{q_{i \alpha}}$ the factorization of $Q$ on $\mathfrak{X}_{\alpha}$. Since the family of extensions of $\mathfrak{X}_{\alpha}$ is cofinal in the collection of all $\mathfrak{X}_{\beta}$, to define $Q_{i}$ it suffices to define $Q_{i \beta}$ on extensions $\mathfrak{X}_{\beta}$ in a consistent fashion. Since there is no splitting or coalescing upon restriction to $\mathfrak{X}_{\alpha}$, we have a unique factorization $Q_{\beta}=Q\left(x_{0}\right) \prod Q_{i \beta}^{q_{i \beta}}$ on $\mathfrak{X}_{\beta}$ with $q_{i \beta}=q_{i \alpha}, Q_{i \beta} \mid \mathfrak{X}_{\alpha}=Q_{i \alpha}$; the uniqueness guarantees consistency. Clearly $Q=Q\left(x_{0}\right) \prod Q_{i}^{q_{i}}$ since this holds on each subspace $\mathfrak{X}_{\beta}$, and the $Q_{i}$ are irreducible since they are on $\mathfrak{X}_{\alpha}$. Note that the factorization of $Q$ takes the same form as that of $Q_{\alpha}$.

To prove uniqueness of this factorization, as usual it is enough to prove irreducible forms are prime. If $P$ is irreducible, by what we just noted it must be irreducible on any stability space. Suppose $P$ is not prime, say $P$ divides $R S$ but not $R$ or $S$; then $R / P$ and $S / P$ are not polynomials, hence there are subspaces $\mathfrak{X}_{\rho}, \mathfrak{X}_{\sigma}$ where they cannot be represented by polynomials. If $\mathfrak{X}_{\pi}$ is a stability space for $P$ containing $\mathfrak{X}_{\rho}$ and $\mathfrak{X}_{\sigma}$ then $P_{\pi}$ divides $R_{\pi} S_{\pi}$ but not $R_{\pi}$ or $S_{\pi}$; but $P_{\pi}$ is irreducible, hence prime, and this is a contradiction.

TheOREM 2 (Hilbert Nullstellensatz). If $\Phi$ is algebraically closed, $P$ and $Q$ forms with $P(x)=0 \Rightarrow Q(x)=0$ then $P$ is composed of irreducible factors of $Q$.

Proof. If $P=\prod P_{i}^{p_{i}}$ for $P_{i}$ irreducible it suffices to prove $P_{i}$ divides $Q$. If not, there would be a space $\mathfrak{X}_{\alpha}$ where $Q / P_{i}$ cannot be represented as a polynomial. Choosing a stability space $\mathfrak{X}_{\pi}$ for $P_{i}$ containing $\mathfrak{X}_{\alpha}$ we observed in the last theorem that $P_{i \pi}$ is irreducible. But for $x \in \mathfrak{X}_{\pi} P_{i \pi}(x)=0 \Rightarrow P_{i}(x)=0 \Rightarrow P(x)=0 \Rightarrow Q(x)=0$ $\Rightarrow Q_{\pi}(x)=0$, so by the ordinary Nullstellensatz for $\mathfrak{X}_{\pi} P_{i \pi}$ divides $Q_{\pi}$, which is impossible since $\mathfrak{X}_{\pi}$ contains $\mathfrak{X}_{\alpha}$.

We now apply these results to situations involving "automorphic forms." $Q$ will denote a form on $\mathfrak{Y}$ with factorization $Q=Q\left(y_{0}\right) \prod Q_{i}^{q_{i}}$.

LEMMA 2 [4, p. 944]. If $Q$ admits composition with a rational mapping $E: x \rightarrow E_{x}$ of $\mathfrak{X}$ into $\operatorname{Hom}(\mathfrak{Y}, \mathfrak{Y})$,

$$
Q\left(E_{x} y\right)=e(x) Q(y)
$$

for some nonzero rational function $e$ on $X$ and for all $x \in \mathfrak{X}, y \in \mathfrak{Y}$ where both sides are defined, then there are rational functions $e_{i}$ and a permutation $\pi=\pi(E)$ such that

$$
Q_{i}\left(E_{x} y\right)=e_{i}(x) Q_{\pi(i)}(y) .
$$


In particular, if $E_{c}=I$ for some $c \in \mathfrak{X}$ then $\pi(E)=1$ and each irreducible factor admits composition with $E$ :

Proof. We have

$$
Q_{i}\left(E_{x} y\right)=e_{i}(x) Q_{i}(y)
$$

$$
Q\left(y_{0}\right) \prod Q_{i}\left(E_{x} y\right)^{q_{i}}=e(x) Q\left(y_{0}\right) \prod Q_{i}(y)^{q_{i}} .
$$

Both sides may be regarded as polynomial functions on $\mathfrak{Y}$ with coefficients in the field $\mathscr{R}(\mathfrak{X})$. The $Q_{i} \circ E_{x}$ are still forms on $\mathfrak{Y}$ of the same degree as $Q_{i}$ since $E_{x}$ is linear, so none are constant, and hence if one of the $Q_{i} \circ E_{x}$ split into proper factors the factorization on the left side would have a greater total number of prime factors than that on the right. This is impossible, so each $Q_{i} \circ E_{x}$ is irreducible as a polynomial in $y$. Since the primes in a factorization are unique up to a constant multiple,

as desired.

$$
Q_{i}\left(E_{x} y\right)=e_{i}(x) Q_{\pi(i)}(y)
$$

We next generalize a result of $\mathrm{H}$. Braun and $\mathrm{M}$. Koecher $[1, \mathrm{p} .81]$. Let $\mathfrak{Y}(Q)$ $=\{y \mid Q(y) \neq 0\}$ and $\mathscr{G}(Q)$ the group of invertible linear transformations on $\mathfrak{Y}$ sending $\mathfrak{Y}(Q)$ onto itself.

LEMMA 3. If $\Phi$ is algebraically closed and $W \in \mathscr{G}(Q)$ then there are scalars $q_{i}(W) \in \Phi$ and a permutation $\pi=\pi(W)$ such that

$$
Q_{i}(W y)=q_{i}(W) Q_{\pi(i)}(y) .
$$

Thus the reduced form $Q_{0}=\prod Q_{i}$ admits composition with $\mathscr{G}(Q)$,

$$
Q_{0}(W y)=q_{0}(W) Q_{0}(y) .
$$

$W \rightarrow q_{0}(W)$ and $W \rightarrow \pi(W)$ are a homomorphism and antihomomorphism of $\mathscr{G}(Q)$ into $\Phi$ and the symmetric group respectively, which are continuous in the sense that if $E: x \rightarrow E_{x}$ is a rational mapping of $\mathfrak{X}$ into $\mathscr{G}(Q) \subset \operatorname{Hom}(\mathfrak{Y}, \mathfrak{Y})$ then $q_{0}(x)=q_{0}\left(E_{x}\right)$ is a rational function on $\mathfrak{X}$ and $\pi(x)=\pi\left(E_{x}\right)$ is constant.

Proof. By definition of $\mathscr{G}(Q), Q(W y)=0 \Leftrightarrow Q(y)=0$, so by the Hilbert Nullstellensatz $Q(W y)=Q\left(y_{0}\right) \prod Q_{i}(W y)^{q_{i}}$ and $Q(y)=Q\left(y_{0}\right) \prod Q_{i}(y)^{q_{i}}$ have the same irreducible factors. Since $W$ is invertible, the $Q_{i}(W y)$ are still distinct and irreducible, so they coincide with the $Q_{i}(y)$ up to order and constant multiples:

$$
Q_{i}(W y)=q_{i}(W) Q_{\pi(i)}(y) .
$$

The statements about $Q_{0}, q_{0}, \pi$ are clear. If $E: \mathfrak{X} \rightarrow \mathscr{G}(Q)$ is a rational mapping then $q_{0}(x)=q_{0}\left(E_{x}\right)=Q_{0}\left(E_{x} y_{0}\right)$ is a rational function, and by Lemma 2 there is a permutation $\pi=\pi(E)$ with $Q_{i}\left(E_{x} y\right)=e_{i}(x) Q_{\pi(i)}(y)$ for the irreducible factors of $Q_{0}$ (which are the same as the irreducible factors of $Q$ ). Thus $e_{i}(x)=q_{i}(x)$ and $\pi(E)$ $=\pi\left(E_{x}\right)$ is independent of $x$.

Let us recall another result along these lines. 
LEMMA 4 [4, p. 948]. If $E: x \rightarrow E_{x}$ is a rational mapping of $\mathfrak{X}$ into $\operatorname{Hom}(\mathfrak{Y}, \mathfrak{Y})$ with $E_{c}=I$ for some $c \in \mathfrak{X}$ and

for all $x$ then

$$
\partial \log Q \circ E_{x}=\partial \log Q
$$

$$
Q=Q^{\prime} Q^{\prime \prime}
$$

where all the irreducible factors of $Q^{\prime}$ admit composition with $E$ and where $Q^{\prime \prime}$ is inseparable.

The proof in [4] depended only on the factorization theorem, hence carries over to this setting.

3. Generically algebraic algebras. An algebra $\mathfrak{A}$ on the vector space $\mathfrak{X}$ is called generically algebraic if

(i) $\mathfrak{A}$ is a power-associative algebra with identity

(3) (ii) there is a monic polynomial $f_{x}(\lambda) \in \mathscr{P}(\mathfrak{X})[\lambda]$ satisfied by every element $x$ of $\mathfrak{A}: f_{x}(x)=0$.

Note that by power-associativity $f_{x}(x)$ makes sense. The generic minimum polynomial $m_{x}(\lambda)=\sum m_{i}(x) \lambda^{i}, m_{i}(x) \in \mathscr{P}(\mathfrak{X})$ is the monic polynomial of least degree satisfying $m_{x}(x)=0$ identically.

THEOREM 3. $m_{x}(\lambda)$ divides any polynomial $p_{x}(\lambda) \in \mathscr{R}(\mathfrak{X})[\lambda]$ for which $p_{x}(x)=0$ identically where defined.

Proof. By power-associativity, $\Phi[x]$ is a commutative-associative algebra with identity for each $x \in \mathfrak{A}$, so for any $f, g \in \Phi[\lambda]$ we have $f(x) g(x)=(f g)(x)$. Thus the set of $p_{x}(\lambda)$ in the theorem forms an ideal in $\mathscr{R}(\mathfrak{X})[\lambda]$; since $\mathscr{R}(\mathfrak{X})$ is a field this ideal is principal, so let $p_{x}(\lambda)$ be its (unique) monic generator. It suffices to prove $m_{x}(\lambda)$ $=p_{x}(\lambda)$.

We have

$$
m_{x}(\lambda)=p_{x}(\lambda) q_{x}(\lambda)
$$

for some monic $q_{x}(\lambda) \in \mathscr{R}(\mathfrak{X})[\lambda]$. If $\mathfrak{X}_{\alpha}$ is a finite-dimensional subspace which has nonempty intersection with the domains of each of the coefficients of $p_{x}(\lambda)$ and $q_{x}(\lambda)$ then upon restriction we get

$$
m_{x \alpha}(\lambda)=p_{x \alpha}(\lambda) q_{x \alpha}(\lambda)
$$

for $m_{x \alpha}(\lambda) \in \mathscr{P}\left(\mathfrak{X}_{\alpha}\right)[\lambda]$ and $p_{x \alpha}(\lambda), q_{x \alpha}(\lambda) \in \mathscr{R}\left(\mathfrak{X}_{\alpha}\right)[\lambda]$. Since $\mathscr{R}\left(\mathfrak{X}_{\alpha}\right)$ is the quotient field of $\mathscr{P}\left(\mathfrak{X}_{\alpha}\right)$ the usual proof involving Gauss' Lemma shows

$$
m_{x \alpha}(\lambda)=p_{x \alpha}^{\prime}(\lambda) q_{x \alpha}^{\prime}(\lambda)
$$

where $p_{x \alpha}^{\prime}(\lambda)=p_{\alpha}(x) p_{x \alpha}(\lambda), q_{x \alpha}(\lambda)=q_{\alpha}(x) q_{x \alpha}^{\prime}(\lambda) \in \mathscr{P}\left(\mathfrak{X}_{\alpha}\right)[\lambda]$ for $p_{\alpha}(x), q_{\alpha}(x) \in \mathscr{R}\left(\mathfrak{X}_{\alpha}\right)$; since $m_{x \alpha}(\lambda)$ is monic, $p_{x \alpha}^{\prime}(\lambda)$ and $q_{x \alpha}^{\prime}(\lambda)$ can be chosen to be monic too. But then since $p_{x \alpha}(\lambda)$ and $q_{x \alpha}(\lambda)$ are also monic we see $p_{\alpha}(x)=q_{\alpha}(x)=1$, and $p_{x \alpha}(\lambda)=p_{x \alpha}^{\prime}(\lambda)$, 
$q_{x \alpha}(\lambda)=q_{x \alpha}^{\prime}(\lambda)$ are already in $\mathscr{P}\left(\mathfrak{X}_{\alpha}\right)[\lambda]$. Since this holds on the cofinal subset of all such $\mathfrak{X}_{\alpha}$ we have $p_{x}(\lambda), q_{x}(\lambda) \in \mathscr{P}(\mathfrak{X})[\lambda]$. By the definition of $m_{x}(\lambda)$ we then have $m_{x}(\lambda)=p_{x}(\lambda)$.

As a corollary we derive the important result that if $m$ is the degree of the generic minimum polynomial there exist elements of degree $m$. For suppose $x_{0}$ is an element of maximal degree $n \leqq m$. We will construct a monic polynomial $n_{x}(\lambda)=\sum n_{i}(x) \lambda^{i}$ of degree $n$ with rational coefficients $n_{i}(x)$ which is satisfied by all elements $x$. By the theorem, this will imply $m_{x}(\lambda)$ divides $n_{x}(\lambda)$, hence $m \leqq n$, and so $m=n$ as desired.

We define a vector space $\mathfrak{Y}$ as the $n$th exterior product of $\mathfrak{X}$,

$$
\mathfrak{Y}=\mathfrak{X} \wedge \cdots \wedge \mathfrak{X}, \quad n \text { times, }
$$

and define polynomial maps $F_{i}: \mathfrak{X} \rightarrow \mathfrak{Y}$ for $i=0,1, \ldots, n$ by

$$
\begin{gathered}
F_{i}(x)=1 \wedge \cdots \wedge x^{i-1} \wedge x^{n} \wedge x^{i+1} \wedge \cdots \wedge x^{n-1} \quad(0 \leqq i \leqq n-1) \\
F_{n}(x)=1 \wedge \cdots \wedge x^{n-1} .
\end{gathered}
$$

Now $F_{n}\left(x_{0}\right) \neq 0$ since by assumption $1, x_{0}, \ldots, x_{0}^{n-1}$ are linearly independent. It will suffice to define the functions $n_{i}(x)$ on the dense set $F_{n}(x) \neq 0$, and here we have a unique expression $x^{n}=-\sum_{i=0}^{n-1} n_{i}(x) x^{i}$ for $n_{i}(x) \in \Phi$ since such $x$ are of degree $n$. Using this expression for $x^{n}$ we see

$$
F_{i}(x)=-n_{i}(x) F_{n}(x)
$$

and hence the $n_{i}(x)$, as "quotients" of two polynomial maps, are rational functions. By definition of $n_{i}(x)$ each element $x$ satisfies

$$
n_{x}(\lambda)=\lambda^{n}+\sum_{i=0}^{n-1} n_{i}(x) \lambda^{i} .
$$

Let us observe a few simple facts about generically algebraic algebras. First, the constant term $m_{0}(x)$ of $m_{x}(\lambda)$ is not identically zero. If it were, $m_{x}(\lambda)=\lambda m_{x}^{\prime}(\lambda)$ where $m_{x}^{\prime}(\lambda)$ is a monic polynomial of lesser degree than $m_{x}(\lambda)$; we will contradict the minimality of $m_{x}(\lambda)$ by showing $m_{x}^{\prime}(x)=0$ for each $x \in \mathfrak{A}$. Consider the finitedimensional commutative-associative algebra $\Phi[x] \subset \mathfrak{A}$. Since $1 \in \Phi[x]$, the set of nonsingular elements $y$ is dense in $\Phi[x]$. But for such $y, y m_{y}^{\prime}(y)=m_{y}(y)=0$ implies $m_{y}^{\prime}(y)=0$; since this polynomial relation holds for all $y$ in a dense set it holds everywhere on $\Phi[x]$, and $m_{x}^{\prime}(x)=0$.

Next, the generic minimum polynomial of an extension algebra $\mathfrak{A}_{\Omega}$ is just the natural extension of the generic minimum polynomial of $\mathfrak{A}$. Indeed, since $\Phi$ is infinite the relation $m_{x}(x)=0$ remains valid upon extension, so $\mathfrak{A}_{\Omega}$ is generically algebraic with minimum polynomial $p_{x}(\lambda)$ which divides the extension $m_{x \Omega}(\lambda)$ of $m_{x}(\lambda)$. On the other hand, if $\omega^{*}: \Omega \rightarrow \Phi$ is a $\Phi$-linear functional with $\omega^{*}(1)=1$ then $\omega^{*} p_{x}(\lambda)$ is a monic $\Phi$-polynomial of the same degree as $p_{x}(\lambda)$, and for $x \in \mathfrak{A}\left\{\omega^{*} p_{x}\right\}(x)$ 
$=\omega^{*}\left\{p_{x}(x)\right\}=0$, so $\omega^{*} p_{x}(\lambda)$ is divisible by $m_{x}(\lambda)$. Thus $m_{x \Omega}(\lambda)$ has degree less than or equal to that of its divisor $p_{x}(\lambda)$, and $m_{x \Omega}(\lambda)=p_{x}(\lambda)$.

Subalgebras and quotient algebras of generically algebraic algebras are again of this type, although with different minimum polynomials. The direct sum $\mathfrak{A}$ $=\mathfrak{U}^{(1)} \oplus \cdots \oplus \mathfrak{A}^{(n)}$ of a finite number of such algebras is again such, with minimum polynomial $m_{\left(x_{1}, \ldots, x_{n}\right)}(\lambda)=m_{x_{1}}^{(1)}(\lambda) \cdots m_{x_{n}}^{(n)}(\lambda)$ the product of the individual minimum polynomials. If $\varphi: \mathfrak{A} \rightarrow \mathfrak{B}$ is a bijection which is a homomorphism on each $\Phi[x]$ for $x \in \mathfrak{A}$ then $\mathfrak{B}$ is generically algebraic with minimum polynomial $m_{\varphi(x)}(\lambda)=m_{x}(\lambda)$. This holds in particular if $\varphi$ is an isomorphism or anti-isomorphism, or more generally an isomorphism of $\mathfrak{A}^{+}$onto $\mathfrak{B}^{+}$. Another case is when $\varphi$ is the identity mapping of $\mathfrak{A}$ into its mutation $\mathfrak{A}^{(\mu)}$, where multiplication in $\mathfrak{A}^{(\mu)}$ for $\mu \in \Phi$ is given by $x \cdot{ }_{\mu} y=\mu x y+(1-\mu) y x$. Thus all mutations have the same generic minimum polynomial; note that $\mathfrak{A}=\mathfrak{U}^{(1)}$ and $\mathfrak{A}^{+}=\mathfrak{A}^{(1 / 2)}$ are particular examples of mutations.

The basic source of generically algebraic algebras is the fact that every finitedimensional power-associative algebra with identity (over $\Phi$ ) is generically algebraic. Indeed, the characteristic polynomial $f_{x}(\lambda)=\operatorname{det}\left(\lambda I-L_{x}\right)$ of the linear transformation $L_{x}$ (=left multiplication by $x$ ) is a monic polynomial in $\lambda$ whose coefficients are polynomial functions of $x$, and from the facts $f_{x}\left(L_{x}\right)=0$ and $L_{x}^{i}(1)=x^{i}$ we have $f_{x}(x)=f_{x}\left(L_{x}\right)(1)=0$. Thus $f_{x}(\lambda)$ satisfies all the requirements of the definition (3); of course in general $f_{x}(\lambda)$ will not be the minimum polynomial.

We give two examples of generically algebraic algebras which are not finitedimensional. If $\Phi$ is of characteristic different from 2 , let $\mathfrak{A}$ be the Jordan algebra of a symmetric bilinear form $(, \quad)$ on an infinite-dimensional space $\mathfrak{X}_{0}$. Here $\mathfrak{X}=\Phi 1+\mathfrak{X}_{0}$, where 1 acts as the identity and multiplication of elements of $\mathfrak{X}_{0}$ is given by $x_{0} \cdot y_{0}=\left(x_{0}, y_{0}\right) 1$. The generic minimum polynomial is $m_{x}(\lambda)=\lambda^{2}-t(x) \lambda$ $+n(x) 1$ where $t\left(\alpha 1+x_{0}\right)=2 \alpha$ and $n\left(\alpha 1+x_{0}\right)=\alpha^{2}-\left(x_{0}, x_{0}\right)$. If the form is nondegenerate, $\mathfrak{A}$ is actually simple. In the other example, assume $\Phi$ has characteristic $p \neq 0$ and let $\mathfrak{A}=\Omega$ be an infinite purely inseparable extension of $\Phi$ of exponent $e$, so $\omega^{p^{e}} \in \Phi$ for all $\omega \in \Omega$. Then the generic minimum polynomial is $m_{x}(\lambda)=\lambda^{p^{e}}-n(x)$ where, for a basis $\left\{\omega_{\alpha}\right\}$ of $\Omega$ over $\Phi$ with $\omega_{\alpha}^{p e}=\varphi_{\alpha}, n(x)$ is given for $x=\sum \xi_{\alpha} \omega_{\alpha}$ by $n(x)=\sum \varphi_{\alpha} \xi_{\alpha}^{p e}$.

4. The generic norm. Throughout this section $\mathfrak{A}$ denotes a generically algebraic algebra with generic minimum polynomial

$$
m_{x}(\lambda)=\sum_{i=0}^{m} m_{i}(x) \lambda^{i}
$$

The generic norm is $N(x)=(-1)^{m} m_{0}(x)$ and the generic trace is $\tau(x)=-m_{m-1}(x)$.

THEOREM 4 [2], [3], [4], [9]. We have the properties

(i) the $m_{i}(x)$ are forms of degree $m-i$;

(ii) the $m_{i}(x)$ are Lie-invariant: $\left.\partial_{D x} m_{i}\right|_{x}=0$ for any derivation $D$ of $\mathfrak{A}$; 
(iii) $m_{i}(1)=(-1)^{m-i}\left(\begin{array}{c}m \\ i\end{array}\right)$;

(iv) $N(\lambda 1-x)=m_{x}(\lambda)$;

(v) $N(x y)=N(x) N(y)$ if $x, y \in \Phi[z]$

(vi) $m_{x}(\lambda)$ has the same irreducible factors as the minimum polynomial $\mu_{x}(\lambda)$ for any $x \in \mathfrak{A}$;

(vii) if $\mathfrak{A}$ is alternative then $N(x y)=N(x) N(y)$ for all $x, y \in \mathfrak{A}$;

(viii) if $\mathfrak{A}$ is a noncommutative Jordan algebra then $N\left(U_{x} y\right)=N(x)^{2} N(y)$ for all $x, y \in \mathfrak{A}$;

(ix) if $\mathfrak{B}$ is a subalgebra of $\mathfrak{A}$ then the restriction $\left.N\right|_{\mathfrak{B}}$ of $N$ to $\mathfrak{B}$ has the same irreducible factors as the generic norm $N_{\mathfrak{B}}$ of $\mathfrak{B}$;

(x) if $M$ is any irreducible factor of $N$ normalized so that $M(1)=1$ then $M(x y)$ $=M(x) M(y)$ when $x, y$ are contained in some associative subalgebra $\mathfrak{B}$ of $\mathfrak{A}$.

Proof. For $0 \neq \alpha \in \Phi$ the polynomial $m_{x}^{(\alpha)}(\lambda)=\alpha^{-m} m_{\alpha x}(\alpha \lambda)=\sum m_{i}(\alpha x) \alpha^{i-m} \lambda^{i}$ is a monic polynomial of degree $m$ satisfying $m_{x}^{(\alpha)}(x)=\alpha^{-m} m_{\alpha x}(\alpha x)=0$, so by Theorem 3 $m_{x}^{(\alpha)}(\lambda)=m_{x}(\lambda)$, hence

$$
m_{i}(\alpha x)=\alpha^{m-i} m_{i}(x)
$$

is a homogeneous polynomial function of degree $m-i$. To prove Lie-invariance we first show

$$
\left.\partial_{D x} x^{i}\right|_{x}=D x^{i}
$$

for any derivation $D$. This is trivially true for $x^{0}=1$, and by induction $\left.\partial_{D x} x^{i+1}\right|_{x}$ $=\left\{\left.\partial_{D x} x\right|_{x}\right\} \cdot x^{i}+x \cdot\left\{\left.\partial_{D x} x^{i}\right|_{x}\right\}=D x \cdot x^{i}+x \cdot D x^{i}=D x^{i+1}$. To show

$$
m_{x}^{\prime}(\lambda)=\sum\left\{\left.\partial_{D x} m_{i}\right|_{x}\right\} \lambda^{i}=0
$$

it suffices to show $m_{x}^{\prime}(x)=0$ since $\left.\partial_{D x} m_{m}\right|_{x}=\left.\partial_{D x} 1\right|_{x}=0$ means $m_{x}^{\prime}(\lambda)$ has degree less than $m$. But $m_{x}^{\prime}(x)=\left.\partial_{D x}\left\{\sum m_{i}(x) x^{i}\right\}\right|_{x}-\left.\sum m_{i}(x) \partial_{D x} x^{i}\right|_{x}=\left.\partial_{D x}\left\{m_{x}(x)\right\}\right|_{x}-D\left\{m_{x}(x)\right\}$ $=0$.

Now fix $x \in \mathfrak{A}$ and consider the commutative-associative ring $\mathfrak{R}=\Phi[x]$. If $\lambda$ is an indeterminate, $\Omega=\Phi(\lambda)$ and $p(x)$ a polynomial in $x$, we can evaluate $N$ at the element $y=(\lambda 1-x) p(x) \in \mathfrak{A}_{\Omega}$ giving a polynomial

$$
m_{x}^{\prime}(\lambda)=N((\lambda 1-x) p(x))
$$

of degree $m$ in $\lambda$ with leading coefficient $N(p(x))$. Since $m_{y}(y)=0$ holds on the extension $\mathfrak{A}_{\Omega}$ we have

$$
\begin{aligned}
m_{x}^{\prime}(\lambda)=N(y) & =(-1)^{m} m_{0}(y)=(-1)^{m}\left\{-\sum_{i=1}^{m} m_{i}(y) y^{i}\right\} \\
& =-(-1)^{m} \sum_{i=1}^{m} m_{i}((\lambda 1-x) p(x))\{(\lambda-x) p(x)\}^{i}
\end{aligned}
$$


where $m_{x}^{\prime}(\lambda)$ and $m_{i}((\lambda 1-x) p(x))$ are in $\Phi[\lambda]$ and $\{(\lambda-x) p(x)\}^{i}$ in $\Re[\lambda]$. Regarding this as a relation in $\Re[\lambda]$ and substituting for $\lambda$ the element $x$ of $\Re$, the nature of the right-hand side clearly shows

$$
m_{x}^{\prime}(x)=0 .
$$

Since this holds for all fixed $x \in \mathfrak{A}$, Theorem 3 shows

$$
N((\lambda 1-x) p(x))=m_{x}^{\prime}(\lambda)=N(p(x)) m_{x}(\lambda) .
$$

In particular, for $p(x)=1$ we have

$$
N(\lambda 1-x)=N(1) m_{x}(\lambda) .
$$

For $\lambda=0, N(-x)=N(1) m_{0}(x)=(-1)^{m} N(1) N(x)$, hence $N(x)=N(1) N(x)$ since $N$ is homogeneous of degree $m$; we saw in the last section that $N \neq 0$, so

$$
N(1)=1 \text {. }
$$

The previous formula then reduces to

$$
N(\lambda 1-x)=m_{x}(\lambda) \text {. }
$$

Identifying coefficients of $\lambda^{i}$ in $(\lambda-1)^{m}=N(\lambda-1)=m_{1}(\lambda)=\sum m_{i}(1) \lambda^{i}$ yields

$$
m_{i}(1)=(-1)^{m-i}\left(\begin{array}{c}
m \\
i
\end{array}\right)
$$

It suffices to prove (v) over the algebraic closure of $\Phi$ since the generic norm of the extension algebra is the natural extension of the given norm. Here $x=q(z)$ $=\alpha_{0} \Pi\left(\alpha_{i}-z\right), y=r(z)$. By the above we have

$$
N(\alpha 1-z)(p(z))=N(\alpha 1-z) N(p(z))
$$

so by repeated splitting off of factors

$$
\text { (v) } \quad N(x y)=N\left(\alpha_{0} \prod\left(\alpha_{i}-z\right) r(z)\right)=\alpha_{0}^{m} \prod N\left(\alpha_{i}-z\right) N(r(z))=N(x) N(y) .
$$

Consider the minimum polynomial $\mu_{x}(\lambda)$ for the fixed element $x$; for $\Re=\Phi[x]$ as before we have $\mu_{x}(\lambda)=(\lambda-x) p(x, \lambda)$ for some $p(x, \lambda) \in \mathfrak{R}[\lambda]$ since $\mu_{x}(x)=0$. Since $\mu_{x}(\lambda) \in \Phi[\lambda] \subset \Omega$ we have

while

$$
N\left(\mu_{x}(\lambda) 1\right)=\mu_{x}(\lambda)^{m}
$$

$$
N\left(\mu_{x}(\lambda)\right)=N((\lambda-x) p(x, \lambda))=N(\lambda 1-x) N(p(x, \lambda))=m_{x}(\lambda) N(p(x, \lambda))
$$

by (v) since $\lambda-x$ and $p(x, \lambda)$ are in $\Omega[x]$. Thus $m_{x}(\lambda)$ divides $\mu_{x}(\lambda)^{m}$. On the other hand, $\mu_{x}(\lambda)$ clearly divides $m_{x}(\lambda)$ since $m_{x}(x)=0$. This establishes (vi).

We prove (vii) and (viii) together. We know that if $\mathfrak{A}$ is alternative (noncommutative Jordan) then $x y\left(U_{x} y\right)$ is invertible if and only if $x$ and $y$ are, with $(x y)^{-1}=y^{-1} x^{-1}\left(\left(U_{x} y\right)^{-1}=U_{x}^{-1} y^{-1}\right)$ and $L_{x}\left(U_{x}\right)$ nonsingular $[4$, p. 943]. Thus we 
see $L_{x}\left(U_{x}\right)$ for $x$ in $\mathfrak{X}(N)$ are in $\mathscr{G}(N)$ in the notation of Lemma 3. Applying Lemma 3 with $E_{x}=L_{x}\left(E_{x}=U_{x}\right)$, the fact that $L_{1}=I\left(U_{1}=I\right)$ shows $N\left(L_{x} y\right)$ $=n(x) N(y) \quad\left(N\left(U_{x} y\right)=n(x) N(y)\right)$; setting $y=1$ shows $n(x)=N(x) \quad\left(n(x)=N\left(x^{2}\right)\right.$ $\left.=N(x)^{2}\right)$, so

$$
\begin{aligned}
N(x y) & =N(x) N(y), \\
N\left(U_{x} y\right) & =N(x)^{2} N(y) .
\end{aligned}
$$

We have

$$
N(x) \neq 0 \Leftrightarrow x \text { is invertible in } \Phi[x]
$$

since if $x$ is invertible we have $1=N\left(x x^{-1}\right)=N(x) N\left(x^{-1}\right)$ by (v), hence $N(x) \neq 0$, and if $N(x) \neq 0$ we can use $m_{x}(x)=0$ to construct an inverse $x^{-1}=N(x)^{-1} x^{\#}$ where the adjoint $x^{\#}$ is defined by

$$
x^{\#}=-(-1) \sum_{i=1}^{m} m_{i}(x) x^{i-1}
$$

Upon restriction, if $\mathfrak{B}$ is a subalgebra of $\mathfrak{A}$ we have

$$
\left.N\right|_{\mathfrak{B}}(x)=0 \Leftrightarrow N(x)=0 \Leftrightarrow x \text { noninvertible in } \Phi[x] \subset \mathfrak{B} \Leftrightarrow N_{\mathfrak{B}}(x)=0 .
$$

This remains valid over the algebraic closure of $\Phi$, so by the Hilbert Nullstellensatz $\left.N\right|_{\mathfrak{B}}$ and $N_{\mathfrak{B}}$ have the same irreducible factors over the algebraic closure, hence also over $\Phi$, proving (ix).

If $\mathfrak{B}$ is associative then (vii) shows $N_{\mathfrak{B}}(x y)=N_{\mathfrak{B}}(x) N_{\mathfrak{B}}(y)$ for $x, y \in \mathfrak{B}$. By Lemma 2 this holds for all normalized irreducible factors of $N_{\mathfrak{B}}$, hence by (ix) for all normalized irreducible factors of $\left.N\right|_{\mathfrak{B}}$. If $M$ is a normalized irreducible factor of $N$ then $\left.M\right|_{\mathfrak{B}}$ is a product of irreducible factors of $\left.N\right|_{\mathfrak{B}}$ and hence also satisfies

$$
M(x y)=\left.M\right|_{\mathfrak{B}}(x y)=\left.\left.M\right|_{\mathfrak{B}}(x) M\right|_{\mathfrak{B}}(y)=M(x) M(y)
$$

for $x, y \in \mathfrak{B}$.

As a corollary to part (viii) we show that an isotope $\mathfrak{Q}^{(u)}$ of a generically algebraic commutative Jordan algebra $\mathfrak{A}$ with generic norm $N$ is again generically algebraic with generic norm $N^{(u)}=N(u) N$. Recall that for invertible $u$ the isotope $\mathfrak{A}^{(u)}$ has the same vector space structure as $\mathfrak{A}$ and multiplication is defined by

$$
x \cdot{ }_{u} y=x \cdot(u \cdot y)+(x \cdot u) \cdot y-(x \cdot y) \cdot u .
$$

The identity element is $1^{(u)}=u^{-1}$. In general, $U_{w}$ is an isomorphism of $\mathfrak{X}^{\left(U_{w}{ }^{u)}\right.}$ onto $\mathfrak{Q}^{(u)}$ for invertible $w$. For our assertion it suffices to pass to the algebraic closure; here $u$ has an invertible square root $v[3, \mathrm{p} .43]$, so $U_{v}$ is an isomorphism of $\mathfrak{A}^{(u)}$ $=\mathfrak{U}^{\left(v^{2}\right)}=\mathfrak{A}^{\left(U_{v}{ }^{1)}\right.}$ onto $\mathfrak{A}=\mathfrak{Y}^{(1)}$. Thus $\mathfrak{Y}^{(u)}$ is generically algebraic, and we saw in $\S 3$ that corresponding elements under $U_{v}$ have the same generic minimum polynomial and generic norm: $m_{x}^{(u)}(\lambda)=m_{U(v) x}(\lambda), \quad N^{(u)}(x)=N\left(U_{v} x\right)=N(v)^{2} N(x)$ $=N\left(v^{2}\right) N(x)=N(u) N(x)$ from (viii) as desired. 
Note that by Lemma 2 part (viii) holds for any normalized factor of $N$; from this we derive

THEOREM 5 [1, pp. 99-100]. If $\mathfrak{A}$ is a generically algebraic commutative Jordan algebra, $M$ a normalized factor of the generic norm $N$, then

$$
\begin{aligned}
& M\left(\lambda 1+U_{x} y^{2}\right)=M\left(\lambda 1+U_{y} x^{2}\right), \\
& M(x, y)=M(x) M\left(x^{-1}+y\right)=M(y) M\left(y^{-1}+x\right)=M(y, x), \\
& M(x+y)=M(x) M\left(x^{-1}+y^{-1}\right) M(y) .
\end{aligned}
$$

Proof. It suffices to assume $x, y$ invertible. If we set $z=U_{x} y^{2}, w=U_{y} x^{2}$ then $w^{2}=U_{y} U_{x} z$ and hence

$$
\begin{aligned}
M(\lambda 1+z) & =M(x)^{-2} M(y)^{-2} M\left(\lambda U_{y} U_{x} 1+U_{y} U_{x} z\right) \\
& =M(x)^{-2} M(y)^{-2} M\left(\lambda w+w^{2}\right) \\
& =M(x)^{-2} M(y)^{-2} M(w) M(\lambda 1+w) \\
& =M(\lambda 1+w)
\end{aligned}
$$

using (viii) and (x). To prove (ii) it suffices to consider the dense set of squares, and

$$
M\left(x^{2}, y^{2}\right)=M(x)^{2} M\left(x^{-2}+y^{2}\right)=M\left(1+U_{x} y^{2}\right)
$$

is symmetric in $x$ and $y$ by (i). Then (iii) follows by replacing $x$ by $x^{-1}$ in (ii).

Parts (vii) and (viii) admit a converse in terms of the adjoint (as in (4)).

THEOREM 6. If $Q$ is a form on a generically algebraic algebra such that

$$
Q(1)=1 \quad Q\left(x x^{\#}\right)=Q(x) Q\left(x^{\#}\right)
$$

then $Q$ is a product of irreducible factors of the generic norm. This holds in particular if $Q(x y)=Q(x) Q(y)$ or $Q\left(U_{x} y\right)=Q(x)^{2} Q(y)$ for all $x, y \in \Phi[z]$.

Proof. $Q(x) Q\left(x^{\#}\right)=Q\left(x x^{\#}\right)=Q(N(x) 1)=N(x)^{q} Q(1)=N(x)^{q}$ shows $Q(x)=0$ $\Rightarrow N(x)=0$; this remains valid on extension to the algebraic closure, so the result follows from the Hilbert Nullstellensatz. Note that $Q\left(U_{y} x\right)=Q\left(y^{2}\right) Q(x)$ for all $y \in \Phi[x]$ implies the same holds for all $y \in \Omega[x], \Omega$ the algebraic closure of $\Phi$, and if $x$ is nonsingular then so is $x^{\#}$ and it has a square root $y \in \Omega\left[x^{\#}\right] \subset \Omega[x]$, so that we have $Q\left(x x^{\#}\right)=Q\left(U_{y} x\right)=Q\left(y^{2}\right) Q(x)=Q\left(x^{\#}\right) Q(x)$ if $Q$ admits Jordan composition on each $\Phi[z]$.

5. Reduced norms. For any element $x$ we can define its reduced minimum polynomial $\mu_{x}^{0}(\lambda)$ to be the monic polynomial of least degree such that $\mu_{x}^{0}(x)$ is nilpotent. If over the algebraic closure $\Omega$ we have

$$
x=\sum_{i=1}^{d} \alpha_{i} e_{i}+z_{i}
$$


for $\alpha_{i}$ distinct scalars in $\Omega, e_{i}$ orthogonal idempotents and $z_{i}=e_{i} z_{i}$ nilpotent elements in $\Omega[x] \subset \mathfrak{A}_{\Omega}$ then

$$
\mu_{x}^{0}(\lambda)=\prod_{i=1}^{d}\left(\lambda-\alpha_{i}\right)
$$

Corresponding to the definition of the generic minimum polynomial one can define the generic reduced minimum polynomial $m_{x}^{0}(\lambda)=\sum_{i=0}^{m_{0}} m_{i}^{0}(x) \lambda^{i}$ to be the monic polynomial of least degree in $\lambda$ with coefficients which are polynomial functions such that $m_{x}^{0}(x)$ is nilpotent for each $x$. Thus $m_{x}^{0}(\lambda)$ is the least polynomial a power of which is divisible by $m_{x}(\lambda)$. The reduced norm $N_{0}$ is $(-1)^{m_{0}}$ times the constant term and the reduced trace $\tau_{0}(x)$ the negative of the $m_{0}-1$ term of $m_{x}^{0}(\lambda)$. We have

$$
m_{x}^{0}(\lambda)=N_{0}(\lambda 1-x)
$$

and $\mu_{x}^{0}(\lambda), \mu_{x}(\lambda), m_{x}^{0}(\lambda), m_{x}(\lambda)$ all have the same roots. If $N=\prod N_{i}^{n_{i}}$ is the factorization of the generic norm $N$ we see $N_{0}=\prod N_{i} \cdot m_{0}$ is called the reduced degree of $\mathfrak{A}$ over $\Phi$; unlike the generic degree $m$ it depends on the base field. The degree of $\mathfrak{A}$ is the maximal number of pairwise orthogonal idempotents over the algebraic closure.

The discriminant $\delta$ of $\mathfrak{A}$ is the discriminant of $m_{x}^{0}(\lambda)$; it is a homogeneous form of degree $\frac{1}{2} m_{0}\left(m_{0}+1\right)$ such that $\delta(x)=0$ if and only if $m_{x}^{0}(\lambda)$ has a repeated root. We say $\mathfrak{A}$ is unramified over $\Phi$ if $\delta \neq 0$ is not identically zero. Note that this will be true if and only if there are $m_{0}$ pairwise orthogonal idempotents over the algebraic closure, i.e., $\mathfrak{A}$ is unramified over $\Phi$ if and only if the reduced degree of $\mathfrak{A}$ over $\Phi$ equals the degree of $\mathfrak{A}$. Indeed, since $\mu_{x}^{0}(\lambda)$ divides $m_{x}^{0}(\lambda)$ and both have the same roots, $m_{x}^{0}(\lambda)$ will have repeated roots unless $\mu_{x}^{0}(\lambda)=m_{x}^{0}(\lambda)$, in which case by (5), (6) $\mathfrak{A}_{\Omega}$ contains $d=m_{0}$ orthogonal idempotents. Thus if $\mathfrak{A}$ is unramified over $\Phi$ it will remain unramified over any extension field, and the reduced norm on the extension will just be the natural extension of $N_{0}$.

Now the factors $N_{i}$ are distinct irreducible forms, so the $m_{x}^{i}(\lambda)=N_{i}(\lambda 1-x)$ are distinct irreducible polynomials in $\mathscr{P}(\mathfrak{X})[\lambda]$, hence the resultant $R_{i j}$ of $m_{x}^{i}(\lambda)$ and $m_{x}^{j}(\lambda)$ is nonzero. But this resultant is a form $R_{i j}(x)$ of degree $m_{i} m_{j}$. Thus on the dense set where all $R_{i j}(x) \neq 0$ no two $m_{x}^{i}(\lambda)$ have a common root, so the discriminant $\delta(x)$ of $m_{x}^{0}(\lambda)$ vanishes if and only if the discriminant $\delta_{i}(x)$ of some $m_{x}^{i}(\lambda)$ vanishes: $\delta(x)=0 \Leftrightarrow \prod \delta_{i}(x)=0$. Thus as elements of $\mathscr{P}(\mathfrak{X})$

$$
\delta=0 \Leftrightarrow \prod \delta_{i}=0
$$

or $m_{x}^{0}(\lambda)$ is ramified if and only if some $m_{x}^{i}(\lambda)$ is ramified.

6. Normed algebras. In this section we assume $\Phi$ has characteristic different from 2. Recall $[4,5]$ that a normed algebra is a nonassociative algebra $\mathfrak{A}$ on $\mathfrak{X}$ with identity element $c=1$ which carries a norm $Q: Q$ is a form of degree $q>0$ with 
$Q(c)=1$ which admits composition $Q\left(E_{x} y\right)=e(x) Q(y), Q\left(F_{x} y\right)=f(x) Q(y)$, where $E, F \in \mathscr{R}(\mathfrak{X}, \operatorname{Hom}(\mathfrak{X}, \mathfrak{X}))$ satisfy $E_{c}=F_{c}=I,\left.\partial_{u} E\right|_{c}=\alpha L_{u},\left.\partial_{u} F\right|_{c}=\beta R_{u}$ for $0 \neq \alpha, \beta \in \Phi$, and which is nondegenerate in the sense that its trace form $\tau=\tau_{c}$ is a nondegenerate bilinear form (as in (2), $\tau_{c}(u, v)=-\left.\partial_{u} \partial_{v} \log Q\right|_{c}$ ). Every normed algebra is a separable noncommutative Jordan algebra which is a direct sum of a finite number of simple ideals which are either finite-dimensional or of degree 2 over their centers. Under these conditions [5, p. 77] each element satisfies the polynomial

$$
q_{x}(\lambda)=Q(\lambda 1-x) .
$$

Since $Q(c)=1$, this is a monic polynomial function, so we have

THEOREM 7. Every normed algebra is generically algebraic.

Part of the Generalized Schafer Conjecture [5] is that the norm $Q$ of a normed algebra is a product of irreducible factors of the generic norm $N$; Theorems 1 and 7 show that this makes sense, i.e., that we can define $N$ and its irreducible factors. It was established for finite-dimensional $\mathfrak{A}$ in $[4$, p. 949] and for infinite-dimensional algebras in the special cases of forms admitting associative or Jordan composition in $[5$, p. 82]. In this section we will apply our results to settle the general case.

THEOREM 8. If $\mathfrak{A}$ is a normed algebra with norm $Q$ then $Q$ has the same irreducible factors. as the generic norm $N$ of $\mathfrak{A}$.

Proof. As usual it suffices to prove this result over the algebraic closure $\Omega$ of $\Phi$. If $M$ is an irreducible factor of $N$ then $M(x)=0 \Rightarrow N(x)=0 \Rightarrow x$ noninvertible $\Rightarrow Q(x)=0$ since if $Q(x) \neq 0$ we could use $q_{x}(x)=0$ in (7) to construct an inverse. By the Hilbert Nullstellensatz, $M$ is an irreducible factor of $Q$.

Conversely, suppose $P$ is a normalized irreducible factor of $Q$. By Lemma 2 we see $P$ admits composition with $E, F$ since $Q$ does. By [5, p. 75] we have

(iii) $\quad \sigma$ is associative: $\sigma(u v, w)=\sigma(u, v w)$

where $\sigma_{y}(u, v)=-\left.\partial_{u} \partial_{v} \log P\right|_{y}$. If $Q(x), Q(y) \neq 0$ then $x, y$ are invertible, so $U_{x}, U_{y}$ are $[4$, p. 943], hence for $u \in X$

$$
\begin{aligned}
\left.\partial_{u} \log \left\{P \circ U_{x}\right\}\right|_{y} & =\left.\partial_{U(x) u} \log P\right|_{U(x) y} & & \text { (by (1)) } \\
& =\sigma_{U(x) y}\left(U_{x} y, U_{x} u\right) & & \text { (by (i)) } \\
& =\sigma_{U(x) y}\left(U_{U(x) y} U_{x}^{-1} U_{y}^{-1} y, U_{x} u\right) & & \left(U_{U(x) y}=U_{x} U_{y} U_{x}\right) \\
& =\sigma\left(U_{x}^{-1} U_{y}^{-1} y, U_{x} u\right) & & \text { (by(ii)) } \\
& =\sigma\left(U_{y}^{-1} y, u\right) & & \text { (by (iii)) } \\
& =\sigma_{y}(y, u) & & \text { (by (ii)) } \\
& =\left.\partial_{u} \log P\right|_{y} . & & \text { (by (i)) }
\end{aligned}
$$


Since this relation holds on a dense set of $x$,

$$
\partial \log \left\{P \circ U_{x}\right\}=\partial \log P
$$

holds for all $x$. By Lemma 4 with $E_{x}=U_{x}$ we have $P=P^{\prime} P^{\prime \prime}$ where $P^{\prime}$ admits composition with $U_{x}$ and where $P^{\prime \prime}$ is inseparable; since $\Omega$ is perfect, $P^{\prime \prime}$ is a $p$ th power, so by irreducibility of $P$ we know $P=P^{\prime}$ admits $U_{x}: P\left(U_{x} y\right)=p(x) P(y)$. Then $p(x)=P\left(U_{x} 1\right)=P\left(x^{2}\right)$ since $P$ is normalized, and $P\left(U_{x} y\right)=P\left(x^{2}\right) P(y)$. For $y=x^{2}$ this gives $P\left(y^{2}\right)=P(y)^{2}$, and since the set of squares is dense $P\left(x^{2}\right)=P(x)^{2}$ for all $x$. Then $P\left(U_{x} y\right)=P(x)^{2} P(y)$ and we can apply Theorem 6 to conclude $P$ is an irreducible factor of $N$.

7. Homogeneous algebras. In this section we indicate how the geometric approach of H. Braun and M. Koecher [1, Chapters 2-3] can be carried over to the infinite-dimensional case. The concepts introduced will not distinguish between the geometry of $\mathfrak{A}$ and that of $\mathfrak{A}^{+}$. Throughout this section $\mathfrak{A}$ will denote a generically algebraic algebra with generic norm $N$ on a vector space $\mathfrak{X}$ over a field $\Phi$ of characteristic $\neq 2$.

The structure group $\mathscr{G}(\mathfrak{X})$ is the set of invertible linear transformations $W$ on $\mathfrak{X}$ such that

$$
(W x)^{-1}=W^{\#-1} x^{-1}
$$

for some invertible linear transformation $W^{\#}$ and all $x$ where both sides are defined. As usual, $\mathscr{G}(\mathfrak{U})$ is an algebraic group with involution \#.

Now $x \rightarrow x^{-1}$ is a rational mapping, so on the set $\mathfrak{X}(N)=\{x \mid N(x) \neq 0\}$ where it is defined we have a linear transformation

$$
H_{x}=-\left.\partial\left\{x^{-1}\right\}\right|_{x}
$$

In particular,

$$
H_{c}=I \text {. }
$$

The Euler differential equations for the homogeneous mapping $x \rightarrow x^{-1}$ of degree -1 imply

$$
H_{x} x=x^{-1} .
$$

Differentiating the relation $\left(x^{-1}\right)^{-1}=x$ yields $H_{x^{-1}} H_{x}=I$ by the chain rule, and similarly for $x$ replaced by $x^{-1}$, so

$$
H_{x^{-1}}=H_{x}^{-1} .
$$

Differentiating (9) and applying (12) give

$$
W \in \mathscr{G}(\mathfrak{X}) \Leftrightarrow W^{\#} H_{\mathrm{W} x} W=H_{x}
$$

identically in $x$ for some $W^{\#}$. Thus $H_{\mathrm{Wc}}=W^{\#-1} W^{-1}$, so

$$
x=W c \in \mathscr{G}(\mathfrak{U}) c \Rightarrow H_{x} \in \mathscr{G}(\mathfrak{U}) .
$$


The subgroup $\mathscr{H}(\mathfrak{U})$ generated by the $H_{x} \in \mathscr{G}(\mathfrak{Q})$ is a normal subgroup, and applying (14) with $W=H_{x}$, then (12), (13) we see

$$
H_{x}^{\#}=H_{x}
$$

if $H_{x} \in \mathscr{G}(\mathfrak{A})$, so $\mathscr{H}(\mathfrak{A})$ is invariant under the involution.

Applying $\left.\partial_{c}\right|_{x}$ to $x^{-1}$ and to $H_{x} u$ (using commutativity of partials) gives

$$
\begin{gathered}
H_{x} c=x^{-2}, \\
\left.\partial_{c} H_{x}\right|_{x}=-2 L_{x^{-1}}^{+} H_{x} .
\end{gathered}
$$

Differentiating $x x^{-1}=x^{-1} x=1, x^{2} x^{-1}=x^{-1} x^{2}=x$ and averaging yields

$$
R_{x^{-1}}=L_{x} H_{x}, \quad L_{x^{-1}}=R_{x} H_{x}, \quad L_{x^{-1}}^{+}=L_{x}^{+} H_{x},
$$

$$
R_{x^{-1}}\left(L_{x}+R_{x}\right)-L_{x^{2}} H_{x}=L_{x^{-1}}\left(R_{x}+L_{x}\right)-R_{x^{2}} H_{x}=2 L_{x^{-1}}^{+} L_{x}^{+}-L_{x^{2}}^{+} H_{x}=I \text {. }
$$

Applying $\left.\partial_{u}\right|_{c}$ to (19) and using (11),

$$
\left.\partial_{u} H_{x}\right|_{c}=-\left(L_{u}+R_{u}\right)=-2 L_{u}^{+} .
$$

Since $\mathfrak{A}$ is power-associative, $\left\{L_{x^{2}}-R_{x^{2}}+\left(R_{x}-L_{x}\right)\left(L_{x}+R_{x}\right)\right\} y=\left.\partial_{y}\left[x^{2}, x\right]\right|_{x}=0$, so we have a well defined operator

$$
U_{x}=L_{x}\left(L_{x}+R_{x}\right)-L_{x^{2}}=R_{x}\left(R_{x}+L_{x}\right)-R_{x^{2}}=2 L_{x}^{+2}-L_{x^{2}}^{+} .
$$

Thus (20) becomes

$$
U_{x} H_{x}+2 L_{x}^{+}\left[H_{x}, L_{x}^{+}\right]=I .
$$

THEOREM 9. There is a homomorphism $W \rightarrow n(W)$ of $\mathscr{G}(\mathfrak{A})$ into $\Phi$ such that

$$
N(W x)=n(W) N(x) \text {. }
$$

Proof. If $W \in \mathscr{G}(\mathfrak{U})$ then in terms of the adjoint (4) the definition (9) becomes $N(W x)^{-1}(W x)^{\#}=(W x)^{-1}=W^{\#-1} x^{-1}=N(x)^{-1} W^{\#-1} x^{\#}$, so

$$
N(x) W^{\#}(W x)^{\#}=N(W x) x^{\#} .
$$

If we can show that no prime factor $M(x)$ of $N(x)$ divides $x^{\#}$ then $N(x)$ divides $N(W x)$; since $W$ is linear, both are of the same degree in $x$, so

$$
N(W x)=n(W) N(x)
$$

for some $n(W) \in \Phi$ as desired.

So suppose $x^{\#}=M(x) F(x)$ for some polynomial map $F: \mathfrak{X} \rightarrow \mathfrak{X}$. Now $(x-\lambda 1)^{\#}$ is a monic polynomial of degree $m-1$ in $x$ with coefficients in $\Phi[\lambda]$, where $m$ is the degree of $N$. We noted in $\S 3$ that there is an $x \in \mathfrak{X}$ of degree $m$; for such an $x$, $(x-\alpha 1)^{\#}$ is never zero for any scalar $\alpha$ in an extension of $\Phi$. But the polynomial function $M(x-\lambda 1)$ has roots $\alpha$, and for such $\alpha(x-\alpha 1)^{\#}=M(x-\alpha 1) F(x-\alpha 1)$ vanishes, which is a contradiction. 
Corollary. Each $W \in \mathscr{G}(\mathfrak{U})$ maps $\mathfrak{X}(N)$ onto itself.

We say $\mathfrak{A}$ is homogeneous if for some extension $\Omega$ of $\Phi \mathscr{G}\left(\mathfrak{A}_{\Omega}\right)$ acts transitively on $\mathfrak{X}_{\Omega}(N)$.

THEOREM 10 (Equivalence Theorem). The following are equivalent:

(i) $\mathscr{G}\left(\mathfrak{A}_{\Omega}\right)$ is transitive for some $\Omega$,

(ii) $\mathscr{H}\left(\mathfrak{A}_{\Omega}\right)$ is transitive for some $\Omega$,

(iii) $H_{x} \in \mathscr{G}(\mathfrak{U})$ for $x \in \mathfrak{X}(N)$,

(iv) $H_{x} H_{H(x) y} H_{x}=H_{y}$,

(v) $\left(H_{x} y\right)^{-1}=H_{x}^{-1} y^{-1}$,

(vi) $L_{x}^{+}$commutes with $H_{x}$,

(vii) $L_{x}^{+}$commutes with $L_{x^{-1}}^{+}$,

(viii) $U_{x} H_{x}=I$,

(ix) $U_{x} U_{y} U_{x}=U_{U(x) y}$,

(x) $\mathfrak{A}^{+}$is a Jordan algebra.

Proof. (i) $\Leftrightarrow$ (ii) $\Leftrightarrow$ (iii): (i) $\Rightarrow$ (iii) by (15) over $\Omega$ (hence over $\Phi$ ), (iii) $\Rightarrow$ (ii) by (17) since over the algebraic closure every invertible element has a square root, (ii) $\Rightarrow$ (i) is obvious.

(iii) $\Leftrightarrow$ (iv) $\Leftrightarrow$ (v): from (9), (14), (16).

(v) $\Rightarrow$ (vi): applying $\left.\partial_{u}\right|_{c}$ to (v) as a function of $x$ gives $2 H_{y} L_{u}^{+} y=2 L_{u}^{+} y^{-1}$ by (21), hence $2 H_{y} L_{y}^{+}=2 L_{y-1}^{+}=2 L_{y}^{+} H_{y}$ by (19).

(vi) $\Leftrightarrow$ (viii): (vi) $\Rightarrow$ (viii) by (23), (viii) $\Rightarrow$ (vi) since applying $\left.\partial_{c}\right|_{x}$ to (viii) gives $2 L_{x}^{+} H_{x}=2 U_{x} L_{x^{-1}}^{+} H_{x}$ by (18); multiplying on the left by $H_{x}$ and right by $U_{x}$ and then using (viii), (19) gives $2 H_{x} L_{x}^{+}=2 L_{x^{-1}}^{+}=2 L_{x}^{+} H_{x}$.

(vii) $\Leftrightarrow$ (viii): $U_{x} H_{x}=\left(2 L_{x}^{+2}-L_{x^{2}}^{+}\right) H_{x}=2 L_{x}^{+} L_{x^{-1}}^{+}-L_{x^{2}}^{+} H_{x}$ by (19), $I=2 L_{x^{-1}}^{+} L_{x}^{+}$ $-L_{x^{2}}^{+} H_{x}$ by (20).

(vi), (viii) $\Rightarrow(\mathrm{x}): L_{x}^{+}$commutes with $U_{x}^{-1}$, hence $U_{x}$, hence $L_{x^{2}}^{+}$.

(ix) $\Leftrightarrow(x)$ : known result.

(ix) $\Rightarrow$ (iv): it suffices to prove (ix) $\Rightarrow$ (viii); (x) $\Rightarrow$ (vii) is known, hence (ix) $\Rightarrow$ (x) $\Rightarrow$ (vii) $\Rightarrow$ (viii).

As always, $p$ denotes the characteristic of $\Phi \quad$ ( $p$ may be zero).

LEMMA 5 [1, p. 102]. If $\mathfrak{A}$ is homogeneous, $P$ a normalized factor of the generic norm such that $p_{x}(\lambda)=P(\lambda 1-x)$ is a polynomial in $\lambda^{p}$ then $P$ is inseparable. In particular, if $\Phi$ is perfect $P$ is a pth power.

Proof. By the Equivalence Theorem $\mathfrak{A}^{+}$is Jordan, so by Theorem 4 (viii) the generic norm and its factor $P$ admit composition with $U_{x}$. Thus (8.iii) shows the trace form $\sigma$ of $P$ is associative for $\mathfrak{A}^{+}$; for nonsingular $y$,

$$
\begin{aligned}
\sigma\left(u, y^{-1}\right) & =\sigma\left(u, U_{y}^{-1} y\right) \\
& =\sigma_{y}(u, y) \\
& =\left.\partial_{u} \log P\right|_{y} \\
& =\left.P(y)^{-1} \partial_{u} P\right|_{y}
\end{aligned}
$$


so the radical of $\sigma$ is the ideal

$$
\operatorname{Rad} \sigma=\left\{u \mid \partial_{u} P=0\right\} .
$$

But by our assumption that $p_{x}(\lambda)$ is a polynomial in $\lambda^{p}$

$$
0=\frac{d}{d \lambda} p_{x}(\lambda)=\frac{d}{d \lambda} P(\lambda 1-x)=\left.\partial_{1} P\right|_{\lambda 1-x}
$$

Setting $y=-x, \lambda=0$

$$
\left.\partial_{1} P\right|_{y}=0 .
$$

Thus $1 \in \operatorname{Rad} \sigma, \operatorname{Rad} \sigma=\mathfrak{A}, \partial_{u} P=0$ for all $u$, and $P$ is inseparable.

THEOREM 11 [1, p. 105]. If $\mathfrak{A}$ is homogeneous and $\Phi$ is perfect then $\mathfrak{A}$ is unramified over $\Phi$.

Proof. We saw in $\S 5$ it suffices to consider an irreducible factor $P=N_{i}$ of $N$ and show the discriminant $\delta_{i}$ of $p_{x}(\lambda)=P(\lambda 1-x)$ is nonzero. Since $N_{i}$ is irreducible in $\mathscr{P}(\mathfrak{X}), p_{x}(\lambda)$ is irreducible in $\mathscr{P}(\mathfrak{X})[\lambda]$. Let $\mathfrak{X}_{0}$ be a stability space for $\boldsymbol{P}$ with basis $\left\{1=x_{1}, x_{2}, \ldots, x_{n}\right\}$, so if $x_{0}=\xi_{1} x_{1}+\cdots+\xi_{n} x_{n} \in \mathfrak{X}_{0 \Omega}$ for $\Omega=\Phi\left(\xi_{1}, \ldots, \xi_{n}\right)$ then $p_{x_{0}}(\lambda)$ is irreducible in $\Phi\left[\xi_{1}, \ldots, \xi_{n}\right][\lambda]$. If $\delta_{i}=0$ then $\delta_{i}\left(x_{0}\right)=0$, so the irreducible polynomial $p_{x_{0}}(\lambda)$ has repeated roots. This implies it is a polynomial in $\lambda^{p}$, hence $p_{x}(\lambda)$ is too for all $x \in \mathfrak{X}_{0}$. This holds for the cofinal set of all such $\mathfrak{X}_{0}$, so it holds for $p_{x}(\lambda)$ as a polynomial in $\mathscr{P}(\mathfrak{X})[\lambda]$. Since $\Phi$ is perfect, Lemma 5 implies $P$ is a $p$ th power, contradicting the assumed irreducibility.

THEOREM 12 [1, p. 119]. If $\mathfrak{A}$ is homogeneous and $\Phi$ is algebraically closed then the reduced generic minimum polynomial of any primitive idempotent $e$ is

$$
m_{e}^{0}(\lambda)=\lambda^{m_{0}-1}(\lambda-1)
$$

and its reduced trace is

$$
\tau_{0}(e)=1
$$

Every sum $1=e_{1}+\cdots+e_{r}$ of pairwise orthogonal primitive idempotents consists of the same number of elements,

$$
r=m_{0}
$$

If $1=e_{1}+\cdots+e_{r}$ is any sum of pairwise orthogonal primitive idempotents, $x=\sum x_{i j}$ the Peirce decomposition of $x \in \mathfrak{A}$ relative to the $\left\{e_{i}\right\}$ with $x_{i i}=\alpha_{i} e_{i}+z_{i}$ for $z_{i}$ nilpotent, then the reduced trace of $x$ is

$$
\tau_{0}(x)=\sum \alpha_{i} .
$$

Proof. Since $\Phi$ is perfect, $\mathfrak{A}$ is unramified by Theorem 11 ; since $\Phi$ is algebraically closed, we saw in $\S 5$ there are $m_{0}$ orthogonal idempotents (necessarily primitive) $1=f_{1}+\cdots+f_{m_{0}}$. Now $e$ and the $f_{i}$ are primitive, $\mathfrak{A}^{+}$is an algebraic Jordan algebra, and $\Phi$ is algebraically closed, so by [10] the Peirce spaces are 
$\mathfrak{A}_{1}(e)=\Phi e+3, \mathfrak{A}_{1}\left(f_{\mathfrak{i}}\right)=\Phi f_{\mathfrak{i}}+\mathfrak{W}_{\mathfrak{i}}$ for $3, \mathfrak{W}_{i}$ nil subspaces. Thus $U_{e} f_{i}=\alpha_{i} e+z_{i}$, $U_{f_{i}} e=\beta_{i} f_{i}+w_{i}$. Since $e=U_{e} 1=\sum U_{e} f_{i}=\left(\sum \alpha_{i}\right) e+\left(\sum z_{i}\right)$ we know some $\alpha_{i}$ is nonzero. Set $\alpha=\alpha_{i}, z=\alpha^{-1} z_{i}, f=f_{i}, \quad \beta=\beta_{i}, w=\alpha^{-1} w_{i}$, and $g=\alpha^{-1} U_{e} f=e+z, h=\alpha^{-1} U_{f} e$ $=\alpha^{-1} \beta f+w$. By Theorem 5 (i) we know

$$
m_{g}^{0}(\lambda)=N_{0}(\lambda 1-g)=N_{0}(\lambda 1-h)=m_{h}^{0}(\lambda) .
$$

From (5), (6) we see

$$
m_{h}^{0}(\lambda)=\lambda^{m_{0}-1}\left(\lambda-\alpha^{-1} \beta\right) .
$$

Now $N_{0}(\lambda 1-g)=N_{0}(\lambda 1-e) N_{0}\left(1-(\lambda 1-e)^{-1} z\right)=N_{0}(\lambda 1-e)$ by Theorem 4 (x) (since $1, e, z$ generate an associative subalgebra) and the fact that $N_{0}(1-y)=1$ for nilpotent $y$ (since $N_{0}(\lambda 1-y)$ has 0 as its only root $N_{0}(\lambda 1-y)=\lambda^{m_{0}}$ ). Thus

$$
m_{e}^{0}(\lambda)=m_{g}^{0}(\lambda)=m_{h}^{0}(\lambda) .
$$

Since $m_{e}^{0}(\lambda)$ has only the roots 0 and 1 we see $\alpha=\beta$,

In particular,

$$
m_{e}^{0}(\lambda)=\lambda^{m_{0}-1}(\lambda-1) .
$$

$$
\tau_{0}(e)=1 \text {. }
$$

If $1=e_{1}+\cdots+e_{r}$ for $e_{i}$ primitive then

$$
N_{0}\left(\lambda^{r} 1-\lambda^{r-1}\left(e_{1}+\cdots+e_{r}\right)\right)=N_{0}\left(\left(\lambda^{r}-\lambda^{r-1}\right) 1\right)=\left\{\lambda^{r-1}(\lambda-1)\right\}^{m_{0}} .
$$

But since $\Phi e_{1}+\cdots+\Phi e_{r}$ is an associative subalgebra, Theorem 4 (x) shows

$$
N_{0}\left(\lambda^{r} 1-\lambda^{r-1}\left(e_{1}+\cdots+e_{r}\right)\right)=N_{0}\left(\lambda 1-e_{1}\right) \cdots N_{0}\left(\lambda 1-e_{r}\right)
$$

and since the $e_{i}$ are primitive we saw

so

$$
N_{0}\left(\lambda 1-e_{i}\right)=m_{e_{i}}^{0}(\lambda)=\lambda^{m_{0}-1}(\lambda-1)
$$

and hence

$$
\left\{\lambda^{r-1}(\lambda-1)\right\}^{m_{0}}=\left\{\lambda^{m_{0}-1}(\lambda-1)\right\}^{r}
$$

$$
r=m_{0} \text {. }
$$

To calculate $\tau_{0}(x)$, first note that the Peirce spaces are orthogonal under any associative bilinear form, so $\tau_{0}(x)=\sum \tau_{0}\left(x_{i i}\right)$. But $\tau_{0}(z)=0$ for any nilpotent $z$ since $m_{z}^{0}(\lambda)=\lambda^{m_{0}}$. Thus $\tau_{0}\left(x_{i i}\right)=\tau_{0}\left(\alpha_{i} e_{i}+z_{i}\right)=\alpha_{i}$, and

$$
\tau_{0}(x)=\sum \alpha_{i} .
$$

8. Structure theory. Here we will extend the results of $\mathrm{H}$. Braun and $\mathbf{M}$. Koecher [1, Chapter 1] concerning the structure theory of generically algebraic algebras satisfying certain nondegeneracy conditions. Throughout this section $\mathfrak{A}$ denotes a strictly power-associative algebra over a field $\Phi$ of characteristic $\neq 2$.

Since "most" finite-dimensional flexible central simple power-associative algebras carry nondegenerate trace forms, it is reasonable to require as a nondegeneracy condition the existence of enough associative forms to separate points. 
(Indeed, the usual approach to the structure theory of simple algebras over an algebraically closed field constructs a trace form by means of a supplementary family of primitive orthogonal idempotents $\left\{e_{i}\right\}$ as in Theorem 12.) This is a stringent requirement, as is seen by the following

THEOREM 13 [1, p. 32]. If $\mathfrak{A}$ is a strictly power-associative algebra with a family of symmetric associative bilinear forms $\{\sigma\}$ which separates points

$$
\bigcap_{\sigma} \operatorname{Rad} \sigma=0,
$$

then $\mathfrak{A}$ is flexible if the characteristic $\neq 2$ and a noncommutative Jordan algebra if the characteristic $\neq 2,5$.

Proof. One verifies immediately that for any symmetric associative $\sigma$ the function $\sigma([a, b, c], d)$ changes sign under a cyclic permutation:

$$
\sigma([a, b, c], d)=-\sigma([b, c, d], a) .
$$

Hence $\sigma([x, y, x], z)=-\sigma([y, x, z], x)=+\sigma([x, z, x], y)$ is symmetric in $y$ and $z$; but since $\sigma([x, y, x], y)=\sigma([x, y, x], y)+\sigma([y, x, x], y)-\sigma([y, x, x], y)=\sigma([y, x, x]$ $+[x, y, x]+[x, x, y], y)=\sigma\left(\partial_{y}[x, x, x], y\right)=0$ by power associativity, it is also alternating. If the characteristic $\neq 2$ it is identically zero, $[x, y, x] \in \bigcap \operatorname{Rad} \sigma=0$, and $\mathfrak{A}$ is flexible.

By flexibility, to prove $\mathfrak{A}$ is a noncommutative Jordan algebra it suffices to prove $\mathfrak{A}^{+}$is Jordan. Thus we may assume $\mathfrak{A}$ is commutative. In the commutative case

so from (24), (25)

$$
\begin{aligned}
\sigma\left(\left[a^{2}, b, a\right], c\right) & =\sigma\left(\left[a^{2}, a, b\right], c\right)-\sigma\left(\left[a^{2}, a, c\right], b\right) \\
& =\sigma([a, a, b], a c)-\sigma([a, a, c], a b)
\end{aligned}
$$

$$
\begin{aligned}
5 \sigma\left(\left[x^{2}, y, x\right], z\right)= & \left\{\sigma\left(\left[x^{2}, y, x\right], z\right)\right\}+\left\{\sigma\left(\left[x, z, x^{2}\right], y\right)\right\} \\
& +\left\{\sigma\left(\left[x^{2}, x, y\right], z\right)-\sigma\left(\left[x^{2}, x, z\right], y\right)\right\} \\
& +2\{\sigma([x, x, y], x z)-\sigma([x, x, z], x y)\} \\
= & \left\{\sigma\left(\left[x^{2}, y, x\right], z\right)+\sigma\left(\left[x^{2}, x, y\right], z\right)+2 \sigma([x y, x, x], z)\right\} \\
& -\left\{\sigma\left(\left[x^{2}, z, x\right], y\right)+\sigma\left(\left[x^{2}, x, z\right], y\right)+2 \sigma([x z, x, x], y)\right\} \\
= & \sigma\left(\partial_{y}\left[x^{2}, x, x\right], z\right)-\sigma\left(\partial_{z}\left[x^{2}, x, x\right], y\right)=0
\end{aligned}
$$

by power-associativity, so if the characteristic $\neq 2,5 \mathfrak{A}^{+}$is Jordan.

The restriction to characteristic $\neq 5$ is annoying.

A seminormal form on an algebra $\mathfrak{A}$ with identity element is a symmetric associative bilinear function $\sigma: \mathfrak{A} \times \mathfrak{A} \rightarrow \Omega$ with values in some extension field $\Omega$ of $\Phi$ which annihilates nilpotent elements $z$ in any extension of $\mathfrak{A}: \sigma(z)=\sigma(1, z)=0$. The seminormal radical

$$
\mathscr{S}(\mathfrak{R})=\bigcap_{\sigma} \operatorname{Rad} \sigma
$$


is the intersection of the radicals of all seminormal forms $\sigma . \mathfrak{A}$ is seminormal if there are enough seminormal forms to separate points: $\mathscr{S}(\mathfrak{A})=0 . \mathfrak{U} / \mathscr{S}(\mathfrak{U})$ is always seminormal if $\mathfrak{A}$ is algebraic.

If $\tau$ is a seminormal form with values in $\Phi$ then it is called normal, and we have a corresponding normal radical

$$
\mathscr{T}(\mathfrak{A})=\bigcap_{\tau} \operatorname{Rad} \tau .
$$

$\mathfrak{A}$ is normal if the normal forms separate points, $\mathscr{T}(\mathfrak{U})=0$, and again $\mathfrak{U} / \mathscr{T}(\mathfrak{Q})$ is normal if $\mathfrak{A}$ is algebraic. It is easily verified that

$$
\mathscr{S}(\mathfrak{U})=\mathfrak{A} \cap \mathscr{T}(\overline{\mathfrak{A}})
$$

where $\overline{\mathfrak{A}}$ is the extension of $\mathfrak{A}$ to the algebraic closure $\bar{\Phi}$ of $\Phi$.

In this setting Theorem 13 says

THEOREM 14. A seminormal algebra is flexible if the characteristic $\neq 2$ and a noncommutative Jordan algebra if the characteristic $\neq 2,5$.

The usual radical $\mathscr{R}(\mathfrak{U})$ is the maximal nil ideal, and $\mathfrak{A}$ is semisimple if $\mathscr{R}(\mathfrak{U})=0$. By definition a seminormal form annihilates nilpotent elements, so

$$
\mathscr{R}(\mathfrak{U}) \subset \mathscr{P}(\mathfrak{U}) \subset \mathscr{S}(\mathfrak{U}) \subset \mathscr{T}(\mathfrak{U})
$$

where $\mathscr{P}(\mathfrak{R})$ is the set of properly nilpotent elements $z$ such $z, z a, a z$ are nilpotent for all $a \in \mathfrak{A}$. Note that in "pathological" cases $\mathscr{S}(\mathfrak{R})$ and $\mathscr{T}(\mathfrak{K})$ may contain idempotents; indeed, we have $\mathscr{S}(\mathfrak{U})=\mathscr{T}(\mathfrak{l})=\mathfrak{A}$ if $\mathfrak{A}$ is a simple nodal noncommutative Jordan algebra. However, if $\mathscr{S}(\mathfrak{U})$ is nil then $\mathscr{R}(\mathfrak{U})=\mathscr{P}(\mathfrak{H})=\mathscr{S}(\mathfrak{R})$. We saw in the course of Theorem 12 that for a homogeneous algebra the reduced trace $\tau_{0}$ over the algebraic closure is a seminormal form on $\mathfrak{A}^{+}$such that $\operatorname{Rad} \tau_{0}$ is nil (if it contained an idempotent it would contain a primitive one $e$, contradicting $\left.\tau_{0}(e)=1\right)$; we say $\mathfrak{A}$ is strongly homogeneous if $\tau_{0}$ is also associative for $\mathfrak{A}$, in which case we have

THEOREM 15 [1, p. 123]. If $\mathfrak{A}$ is strongly homogeneous then

$$
\mathscr{R}(\mathfrak{U})=\mathscr{P}(\mathfrak{H})=\mathscr{S}(\mathfrak{l}) .
$$

THEOREM $16[1$, p. 54]. A seminormal generically algebraic algebra is a direct sum of a finite number of simple ideals.

Proof. As usual, it suffices to show that each ideal $\mathfrak{B}$ has an identity and is a direct summand of $\mathfrak{A}$ (the number of simple summands will then be bounded by the generic degree of $\mathfrak{A}$ ).

$\mathfrak{B}$ cannot be nil since $\mathscr{S}(\mathfrak{R})=0$, so it contains a principal idempotent $e$ (an increasing chain of idempotents has length at most equal to the generic degree). We have Peirce decompositions

$$
\begin{aligned}
& \mathfrak{U}=\mathfrak{A}_{0}+\mathfrak{A}_{1 / 2}+\mathfrak{A}_{1}, \\
& \mathfrak{B}=\mathfrak{B}_{0}+\mathfrak{B}_{1 / 2}+\mathfrak{B}_{1}
\end{aligned}
$$


where $\mathfrak{B}_{1}=\mathfrak{A}_{1}, \mathfrak{B}_{1 / 2}=\mathfrak{A}_{1 / 2}, \mathfrak{B}_{0}=\mathfrak{B} \cap \mathfrak{A}_{0}$ since $\mathfrak{B}$ is an ideal. Theorem 12 shows $\mathfrak{A}$ is flexible, so $\mathfrak{B}_{0}$ is a subalgebra; since $e$ is principal $\mathfrak{B}_{0}$ is nil, so the orthogonality of Peirce spaces and the fact that a seminormal form $\sigma$ annihilates nil algebras shows $\sigma\left(\mathfrak{B}_{0}, \mathfrak{A}\right)=0, \mathfrak{B}_{0}=0, \sigma\left(\mathfrak{B}_{1 / 2}, \mathfrak{A}\right)=0, \mathfrak{B}_{1 / 2}=\mathfrak{A}_{1 / 2}=0$, and $\mathfrak{B}=\mathfrak{B}_{1}=\mathfrak{A}_{1}$ has identity element $e$ and is a direct summand of $\mathfrak{A}=\mathfrak{A}_{0} \oplus \mathfrak{A}_{1}$.

REMARK. The above theorem actually holds for a slightly larger class of algebras, those which

(i) are strictly power-associative,

(ii) are algebraic,

(iii) are seminormal,

(iv) have chain condition on idempotents.

(One defines $e \geqq f$ if $e f=f e=f$; if $\mathfrak{A}$ is strictly power-associative this is a transitive relation, and the chain condition is equivalent to the nonexistence of an infinite family of pairwise orthogonal idempotents. Note in particular that (iv) follows if the algebra is algebraic of bounded degree.)

Note that an ideal of a seminormal (resp. normal) algebra is again seminormal (resp. normal).

THEOREM 17. If $\mathfrak{A}$ is a simple generically algebraic algebra which is seminormal then $\mathfrak{A}$ is a noncommutative Jordan algebra which is either

(i) a finite-dimensional quasiassociative algebra,

(ii) a finite-dimensional commutative Jordan algebra,

(iii) of degree 2

over its center $\Gamma$. The following are equivalent:

(iv) $\mathfrak{A}$ is normal,

(v) $\mathfrak{A}$ is separable,

(vi) the generic trace $\tau$ is nondegenerate,

(vii) $\mathfrak{A}^{+}$is a normed algebra.

Proof. Let $\bar{\Phi} \supset \Gamma \supset \Sigma \supset \Phi$ where $\bar{\Phi}$ is the algebraic closure of $\Phi, \Gamma$ is purely inseparable over $\Sigma$ of exponent $e$, and $\Sigma$ is separable over $\Phi$ (such a factorization is possible since $\Gamma$ is generically algebraic over $\Phi$ ).

By seminormality we have nonzero seminormal forms $\sigma$ with values in $\bar{\Phi}$ (which are nondegenerate since $\mathfrak{A}$ is simple); each such $\sigma$ has the form $\sigma=\sum \alpha_{i} \sigma_{i}$ for $\alpha_{i} \in \bar{\Phi}$ and $\sigma_{i}$ associative forms with values in $\Phi$ (but not necessarily normal). If we choose $\sigma$ with at least one $\alpha_{i}=1$ and the minimal possible number of nonzero $\alpha_{i}$ then the usual minimality argument shows $\sigma^{g}=\sigma$ for all $\Phi$-automorphisms of $\bar{\Phi}$. This shows that the radical of the extension $\bar{\sigma}$ of $\sigma$ to the normal closure $\bar{\Sigma}$ of $\Sigma$ is invariant under all $\Phi$-automorphisms of $\bar{\Sigma}$. Similarly, if this radical were nonzero it would contain a minimal $x=\sum \alpha_{i} x_{i}$ for $\alpha_{i} \in \bar{\Sigma}$ and $x_{i} \in \mathfrak{A}$ satisfying $x^{g}=x$ for all $g$. But since $\bar{\Sigma}$ is normal this would imply $x \in \mathfrak{A}$, contradicting the nondegeneracy of $\sigma$ on $\mathfrak{A}$. Thus $\mathscr{S}\left(\mathfrak{A}_{\Sigma}\right) \subset \mathscr{S}\left(\mathfrak{A}_{\bar{\Sigma}}\right) \subset \operatorname{Rad} \bar{\sigma}=0$, and $\mathfrak{A}_{\Sigma}$ is seminormal. By Theorem 16 it is a direct sum of simple ideals, and another application of the 
Galois group argument to $\mathfrak{A}_{\Sigma}$ embedded in $\mathfrak{A}_{\bar{\Sigma}}$ shows these are all $\Phi$-isomorphic. It suffices to prove one (hence all) of these is of type (i), (ii), (iii). Such a simple summand is a seminormal generically algebraic algebra over $\Sigma$ with center isomorphic to $\Gamma$.

We have reduced the problem to the case where $\Sigma=\Phi$ and $\Gamma$ is purely inseparable over $\Phi$. We claim in this case any seminormal form is actually $\Gamma$-linear. If $\gamma \in \Gamma$, $\gamma^{p^{e}} \in \Phi, x \in \mathfrak{A}$ then $z=\gamma \otimes x-1 \otimes \gamma x$ is nilpotent in $\mathfrak{A}_{\Gamma}, z^{p^{e}}=0$, so by definition of seminormality $\sigma(z)=0, \gamma \sigma(x)=\sigma(\gamma x)$. Next, we claim $\sigma$ is seminormal on $\mathfrak{A}$ regarded as a $\Gamma$-algebra. If $\Omega \supset \Gamma \supset \Phi,\left\{\omega_{i}\right\}$ a basis for $\Omega$ over $\Gamma, x=\sum \omega_{i} \otimes x_{i} \in$ $\Omega \otimes_{\Gamma} \mathfrak{A}, y=\sum \omega_{i} \otimes x_{i} \in \Omega \otimes_{\oplus} \mathfrak{A}$ then $\sigma(x)=\sigma(y)$. Now $x$ nilpotent $\Rightarrow 0=x^{p^{\prime}}$ $=\sum \omega_{i}^{p^{\prime}} \otimes x_{i}^{p^{\prime}}=\sum \gamma_{i j} \omega_{j} \otimes x_{i}^{p^{\prime}}$ (if $\left.\omega_{i}^{p^{\prime}}=\sum \gamma_{i j} \omega_{j}, \gamma_{i j} \in \Gamma\right) \Rightarrow \sum \gamma_{i j} x_{i}^{p^{\prime}}=0$ for each $j$ $\Rightarrow \sum \gamma_{i j}^{p^{e}} x_{i}^{p^{e+f}}=0$ for each $j\left(\gamma_{i j}^{p e} \in \Phi\right) \Rightarrow y^{p^{e+f}}=0$, and $y$ is nilpotent. By seminormality of $\sigma$ on $\mathfrak{A}$ as a $\Phi$-algebra, $\sigma(y)=0$; but then $\sigma(x)=0$, and $\sigma$ is seminormal on $\mathfrak{A}$ as a $\Gamma$-algebra. Thus $\mathfrak{A}$ is a central-simple seminormal generically algebraic algebra over its center $\Gamma$.

We have reduced the problem to the case where $\Gamma=\Phi$ and $\mathfrak{A}$ is a central-simple seminormal generically, algebraic algebra over its center $\Gamma$. Since the extension of such an algebra remains such (since $\mathscr{S}\left(\mathfrak{H}_{\Omega}\right) \neq \mathfrak{A}_{\Omega}$ we have $\mathscr{S}\left(\mathfrak{A}_{\Omega}\right)=0$ by simplicity, hence the extension remains seminormal), and since a central-simple algebra will be of type (i), (ii), (iii) if and only if its extension is, we may assume $\Phi=\bar{\Phi}$ is algebraically closed. Here we may follow the argument of $[5$, p. 79]; all that was necessary in that argument was that

(a) there is a finite family of primitive supplementary orthogonal idempotents $\left\{e_{i}\right\}_{i \in I}$;

(b) there is a family of symmetric associative bilinear forms $\{\sigma\}$ which separate points;

(c) $\sigma(z)=0$ for $z$ nilpotent;

(d) the Peirce spaces $\mathfrak{A}_{\mathfrak{i t}}=\Phi e_{\mathfrak{i}}+\mathcal{Z}_{\mathfrak{i t}}$ for $\mathcal{B}_{i \mathfrak{i}}^{+}$a nil ideal of $\mathfrak{A}_{\mathfrak{i}}^{+}$;

(e) if $|I| \geqq 3$ then $\mathfrak{A}^{+}$is Jordan.

In our situation (a) is generic algebraicness, (b) and (c) are seminormality, and (d) follows from a

LEMMA 6. If $\lambda \neq 0$ is a linear functional on a power-associative algebra $\mathfrak{A}$ which annihilates nilpotent elements, and if every element of $\mathfrak{A}$ is of the form $a=\alpha 1+z$ for $\alpha \in \Phi, z$ nilpotent, then

$$
\mathfrak{A}=\Phi 1+\mathbb{Z}
$$

for $8=\{z \mid z$ nilpotent $\}=\operatorname{Ker} \lambda$ a nil ideal of $\mathfrak{A}^{+}$.

For the proof of (e) we build up Jordan subalgebras inductively by means of the following

LEMMA 7. If $\mathfrak{A}$ is a commutative strictly power-associative algebra, $\sigma$ a nondegenerate symmetric associative bilinear form on $\mathfrak{A}, 1=e_{1}+e_{2}+e_{3}$ a sum of three 
orthogonal idempotents such that the Peirce subalgebras $\mathfrak{B}_{i j}=\mathfrak{A}_{\mathfrak{i}}+\mathfrak{A}_{\mathfrak{i j}}+\mathfrak{U}_{j j} \quad(i, j$ $=1,2,3)$ are Jordan algebras then $\mathfrak{A}$ itself is a Jordan algebra.

From these we conclude as in [5] that $\mathfrak{A}$ is of type (i), (ii), (iii).

Under these conditions the equivalence of (v), (vi), (vii) in the final assertion of the theorem is well known. Any extension of a normal algebra remains normal, hence semisimple, so (iv) implies (v). Conversely, for separable algebras of types (i), (ii), (iii) every associative form annihilates nilpotent elements (over the algebraic closure it is a linear combination of generic traces), so in this case seminormality and normality are equivalent.

REMARK. In the case of characteristic $\neq 5$ Lemmas 6 and 7 are unnecessary, since (d) and (e) follow from Theorem 13.

COROLLARY. Every normal algebra is strongly homogeneous.

\section{REFERENCES}

1. H. Braun and M. Koecher, Jordan-Algebren, Springer, New York, 1966.

2. N. Jacobson, Some groups of linear transformations defined by Jordan algebras. I, J. Reine Angew. Math. 201 (1959), 178-195.

3. - Generic norm of an algebra, Osaka Math. J. 15 (1963), 25-50.

4. K. McCrimmon, Norms and noncommutative Jordan algebras, Pacific J. Math. 15 (1965), 925-956.

5. - A proof of Schafer's conjecture for infinite dimensional forms admitting composition, J. Algebra 5 (1967), 72-83.

6. R. D. Schafer, On cubic forms permitting composition, Proc. Amer. Math. Soc. 10 (1959), 917-925.

7. - On cubic forms permitting a new type of composition, J. Math. Mech. 10 (1961), 159-174.

8. - On forms of degree n permitting composition, J. Math. Mech. 12 (1963), 777-792.

9. J. Tits, $A$ theorem on generic norms of strictly power associative algebras, Proc. Amer. Math. Soc. 15 (1964), 35-36.

10. K. McCrimmon, Jordan algebras of degree 1, Bull. Amer. Math. Soc. 70 (1964), 702.

Massachusetts Institute of Technology,

Cambridge, Massachusetts 